\title{
Static and dynamic magnetic properties of densely packed magnetic nanowire arrays
}

\author{
O. Dmytriiev, U. A. S. Al-Jarah, P. Gangmei, V. V. Kruglyak, and R. J. Hicken* \\ School of Physics and Astronomy, University of Exeter, Stocker Road, Exeter EX4 4QL, United Kingdom \\ B. K. Mahato, B. Rana, M. Agrawal, and A. Barman \\ Department of Condensed Matter Physics and Material Sciences, S. N. Bose National Centre for Basic Sciences, Block JD, \\ Sector III, Salt Lake, Kolkata 700 098, India \\ M. Mátéfi-Tempfli and L. Piraux \\ Institute of Condensed Matter and Nanosciences, Université catholique de Louvain, Place Croix du Sud, \\ 1, B-1348 Louvain-la-Neuve, Belgium \\ S. Mátéfi-Tempfli \\ Mads Clausen Institute, University of Southern Denmark, Alsion 2, DK-6400 Sønderborg, Denmark
}

(Received 2 December 2012; revised manuscript received 2 April 2013; published 28 May 2013)

\begin{abstract}
The static and dynamic magnetic properties of magnetic nanowire arrays with high packing density $(>0.4)$ and wire diameter much greater than the exchange length have been studied by static and time-resolved magneto-optical Kerr effect measurements and micromagnetic simulations. The nanowires were formed by electrodeposition within a nanoporous template such that their symmetry axes lay normal to the plane of the substrate. A quantitative and systematic investigation has been made of the static and dynamic properties of the array, which lie between the limiting cases of a single wire and a continuous ferromagnetic thin film. In particular, the competition between anisotropies associated with the shape of the individual nanowires and that of the array as a whole has been studied. Measured and simulated hysteresis loops are largely anhysteretic with zero remanence, and the micromagnetic configuration is such that the net magnetization vanishes in directions orthogonal to the applied field. Simulations of the remanent state reveal antiferromagnetic alignment of the magnetization in adjacent nanowires and the formation of vortex flux closure structures at the ends of each nanowire. The excitation spectra obtained from experiment and micromagnetic simulations are in qualitative agreement for magnetic fields applied both parallel and perpendicular to the axes of the nanowires. For the field parallel to the nanowire axes, there is also good quantitative agreement between experiment and simulation. The resonant frequencies are initially found to decrease as the applied field is increased from remanence. This is the result of a change of mode profile within the plane of the array from nonuniform to uniform as the ground state evolves with increasing applied field. Quantitative differences between experimental and simulated spectra are observed when the field is applied perpendicular to the nanowire axes. The dependence of the magnetic excitation spectra upon the array packing density is explored, and dispersion curves for spin waves propagating within the array parallel to the nanowire axis are presented. Finally, a tunneling of end modes through the middle region of the nanowires was observed. The tunneling is more efficient for wires forming densely packed arrays, as a result of the extended penetration of the dynamic demagnetizing fields into the middle of the wires and due to the lowering of the tunneling barrier by the static demagnetizing field of the array.
\end{abstract}

DOI: 10.1103/PhysRevB.87.174429

PACS number(s): 75.75.-c, 75.78.Cd, 75.60.Jk

\section{INTRODUCTION}

Magnetic nanowire arrays (MNWAs) represent a new class of artificial materials not previously seen in nature. They have received considerable attention due to their novel properties and potential applications. Methods for their fabrication, such as nanolithography ${ }^{1,2}$ and electrodeposition ${ }^{2,3}$ into nanoporous templates, for example, track-etched polymer membranes or nanoporous alumina films, have only been developed within the past two decades. They have potential uses as magnetic recording media that overcome the superparamagnetic limit faced by conventional thin film media ${ }^{4,5}$ or as replacements for ferrite-based materials in microwave devices of reduced physical size such as circulators, variable attenuators, phase shifters, modulators, stop-band filters, and power absorbing terminals. ${ }^{6-8}$ The main advantages of MNWA are their low cost of production and well-defined and robust remanent microwave absorption with a frequency that can be tuned through a large range by an appropriate choice of ferromagnetic material. The use of MNWA in microwave circulators that do not require an external bias field has already been demonstrated. ${ }^{9}$ Ferromagnetic nanowires released from the nanoporous template are also good candidates for the application of force or heat to cells and biomolecules and for the delivery of drugs within biomedical science. ${ }^{10,11}$

For effective exploitation of MNWAs it is essential to establish a full understanding of their static and dynamic magnetic properties. Their static magnetic properties have been studied mostly by magnetometry experiments ${ }^{12-15}$ but also by magneto-optical measurements. ${ }^{16-18}$ Their dynamic magnetic properties have been probed by ferromagnetic resonance $^{19-22}$ and Brillouin light scattering. ${ }^{24,25}$ From the 
theoretical point of view, the MNWA is a complicated object. The precessional mode spectrum of isolated magnetic nanowires of finite thickness has been considered analytically in Refs. 26-31. However, with rare exceptions, ${ }^{32}$ theories of the magnetodynamic response of MNWAs are based on the simplifying assumption that individual nanowires can be either treated as macrospins ${ }^{33-35}$ or assumed to be thin compared to the exchange length. Due to the limitations imposed by the required computational resources, dynamic micromagnetic simulations have previously only been performed for single nanowires or large arrays of cylindrical nanodots, ${ }^{36}$ while static simulations have been restricted to arrays of nanowires with diameters of just a few exchange lengths ${ }^{37}$ or short nanowires with length only several times greater than their diameter. ${ }^{13}$

It has been shown experimentally, analytically, and from micromagnetic simulations of a finite number of nanowires $^{35,38}$ and one-dimensional (1D or one-layer) ${ }^{39-41}$ and two-dimensional (2D) $)^{21,33,37}$ arrays that the internal dipolar interaction between nanowires leads to significant modification of the properties of the arrays relative to individual nanowires. It has also been shown that there is a significant difference in the properties of finite arrays and quasi-infinite arrays, for which the lateral dimensions of the array are significantly larger than the length of the nanowires. ${ }^{35,37}$ A theoretical method was developed in Refs. 42 and 43 by which the effect of the magnetodipole interaction upon the structure of the mode spectrum was calculated for isolated magnetic elements using full micromagnetic simulations, but so far this method has not been applied to MNWAs.

For a single nanowire with large aspect ratio (defined as the ratio of the length of the nanowire to its diameter) the easy axis and hence the remanent magnetization are expected to lie parallel to the axis of symmetry. Within the end regions, vortex flux closure structures may form for nanowires with diameter significantly exceeding the exchange length, while a flower state structure may form in nanowires with diameter comparable to the exchange length. ${ }^{13}$ However, some dipolar field is generated by the uncompensated surface charges at the ends and shows a strong variation along the length of the nanowire, decreasing rapidly from the ends towards the middle region. For an array of such nanowires with a large aspect ratio (defined as the ratio of the length of the nanowires to the lateral size of the array) the dipolar field again varies strongly along the length of the nanowires. However, for an array with an aspect ratio that is much smaller than unity, the magnetic charges at the ends of individual nanowires combine to form 2D arrays ("layers") of charges on the top and bottom surfaces of the MNWA. The dipolar field associated with the charged layers penetrates much deeper along the length of the nanowires and becomes finite and nearly uniform in their middle part. As a result, the array acquires an easy plane anisotropy that is associated with the charges on its top and bottom surfaces, very much like a magnetic thin film. However, the dipolar field remains nonuniform near the lateral boundaries of the array as a whole and also in the vicinity of its top and bottom surfaces, where the discrete nature of the charged layers is still important. It is possible to draw an analogy with the shape anisotropy of a cylinder. The easy-axis shape anisotropy of a tall cylinder transforms into easy-plane shape anisotropy as the cylinder is compressed into a disk of small aspect ratio. ${ }^{44,45}$ In contrast with this analogy, the easy-axis and easy-plane anisotropies, associated with the shape of the individual nanowires and of the MNWA as a whole respectively, coexist in a MNWA. It is obvious that the strength of the easy-plane shape anisotropy increases with the packing density. The existence of this easy-plane shape anisotropy was demonstrated through the use of a simple macrospin theoretical model in Ref. 46. The presence of the easy-plane shape anisotropy in MNWAs was also described in Refs. 19-21, in terms of a transition from easy-axis to easyplane shape anisotropy, as the value of the packing density $p$ exceeded a threshold value ( $p=1 / 3$ in Refs. 19-21). The influence of this easy-plane shape anisotropy on the ground state of MNWAs was demonstrated in Ref. 47, where vibrating sample magnetometry (VSM) and magnetic force microscopy (MFM) were used to investigate the distribution of magnetization in two square MNWAs with nanowire aspect ratio $\sim 2$ and nominal packing densities $p=0.2$ and 0.38 . An antiferomagnatic ground state ("checkerboard pattern") was observed within the latter array. In the present study we present a systematic and quantitative investigation of the competition between the shape anisotropies, associated with the shape of the individual nanowires and the shape of the array, and their influence upon the ground state. While the magnetic order depends upon the field history for arrays with low packing densities, the magnetic order is largely independent of field history and the remanent magnetization vanishes in arrays with high packing densities (simulations will be presented for $p \geqslant 0.4$ ). We also study how the competition between the different shape anisotropies influences the dynamic properties of MNWAs.

We present experimental studies of Py nanowire arrays with length of $800 \mathrm{~nm}$, average center-to-center separation (a) of 100 and $150 \mathrm{~nm}$, and nominal packing densities $p=$ 0.38 and 0.41 . The experimental static and dynamic properties were studied by magneto-optical Kerr effect (MOKE) magnetometry and optically stimulated time-resolved (TR) MOKE measurements, respectively. We show that TRMOKE measurements are particularly well suited to the study of such MNWA. There is generally a region of reduced internal field at the end of each nanowire. The optical skin depth, which may be extended by waveguiding within the alumina matrix, is somewhat larger than the length of this region, and so, TRMOKE simultaneously probes localized excitations confined within the end region and delocalized excitations within the main part of the nanowires with similar sensitivity. In fact, it was shown in Refs. 16-18 that ferromagnetic nanowires of few-micron length are semitransparent when embedded in an alumina matrix with high packing density. Very good agreement was found in Ref. 18 between hysteresis loops acquired by VSM and Faraday rotation measurements. Here experimental results are compared with micromagnetic simulations of MNWAs with $p=0.4$, close to the nominal packing density of the measured MNWA, and 0.49 and 0.66 performed with the finite element code NMAG. ${ }^{48}$ The method used for calculating the dipolar field within the array allows the static and dynamic micromagnetic studies to be extended to quasi-infinite arrays of magnetic nanowires so that direct comparison can be made with experimental results. 


\section{DETAILS OF THE EXPERIMENT AND MICROMAGNETIC SIMULATION PROCEDURE}

The supported template fabrication process ${ }^{49-52}$ begins with the anodization of a $\sim 1-\mu$ m-thick Al layer sputtered onto the $\mathrm{Au}(20 \mathrm{~nm}) / \mathrm{Ta}(5 \mathrm{~nm}) / \mathrm{sapphire}$ substrate. Two periodic arrays of $\mathrm{Ni}_{80} \mathrm{Fe}_{20}$ (Py) nanowires were fabricated by electrodeposition of Py into thin-film alumina templates (about $1.1 \mu \mathrm{m}$ thick) using the Au layer at the bottom of the pores as a working electrode for subsequent electrodeposition. The filled template was then thinned down to $0.8 \mu \mathrm{m}$ by mechanical polishing using colloidal silica (siton) in such a way that a very large proportion of the nanowires end at the template surface. The surface was then protected by deposition of $5 \mathrm{~nm}$ of $\mathrm{Al}$. The lengths of the electrodeposited nanowires are almost uniform and equal to the thickness of the template. The average center-to-center separation of the nanowires was found to be $a=150$ and $100 \mathrm{~nm}$ for the first and second arrays shown in Figs. 1(a) and 1(b), respectively. The packing density was estimated by converting the images in Figs. 1 (a1) and 1(b1) to black-and-white bitmaps and using image-processing software to calculate the fraction of white area. Nominal values of $p=0.38$ and 0.41 were obtained for the first and second arrays, respectively. However, there are regions within the SEM images where nanowires appear to be missing. On closer inspection, black holes may be observed against a dark gray background, suggesting that a nanowire is present but does not extend to the polished surface. The calculated packing densities are also sensitive to the grayscale threshold used to generate the black-and-white bitmap. Values of $p$ up to 0.49 and 0.51 could be obtained from Figs. 1(a) and 1(b), respectively, by adjusting the threshold and selecting regions with no missing nanowires. However, the effect of the polishing procedure should also be considered. The pores within the alumina template are known to have smooth edges and cylindrical shape. The irregular shape of the of the nanowire ends within the images is therefore most likely a result of the polishing, and such distortions must be kept in mind when interpreting the associated magnetic properties. If polishing is assumed to reduce the apparent area of the end of the nanowire, then an upper limit for the packing density can be obtained by measuring the minimum edge-to-edge nanowire separations and using these values to infer a diameter of $d=110$ and $85 \mathrm{~nm}$ for the first and second samples, respectively. Calculating the packing densities for an ideal hexagonal close-packed lattice then yields somewhat larger values of $\tilde{p}=0.49$ and 0.66 for the first and second samples, respectively.

Polar and longitudinal MOKE hysteresis loops were acquired to investigate the static magnetization properties of the arrays. Measurements were performed with a continuous wave He-Ne laser of $633 \mathrm{~nm}$ wavelength while a balanced optical bridge detector was used to detect the change of polarization and intensity of the beam reflected from the sample. TRMOKE measurements were made to investigate the optically induced magnetization dynamics and transient reflectivity of the samples. In this particular pump-probe measurement technique, ${ }^{53}$ a Ti:sapphire regenerative amplifier laser system was used to generate pulses of $800 \mathrm{~nm}$ wavelength, at $100 \mathrm{kHz}$ repetition rate, with an intensity autocorrelation of 82 fs that corresponds to a hyperbolic secant pulse shape of 53 fs width. Each pulse was split into a pump and probe part. The pump pulse had energy of up to $0.5 \mu \mathrm{J}$, while the probe pulse was passed through a second harmonic generation crystal so as to provide a pulse of $400 \mathrm{~nm}$ wavelength with fixed energy of $2.5 \mathrm{~nJ}$. The relative time of arrival of pump and probe at the sample was controlled by passing the pump through an optical delay line consisting of a hollow corner cube reflector mounted upon a stepper-motor-controlled translation stage that had $1.25 \mu \mathrm{m}$ resolution and length of $600 \mathrm{~mm}$, corresponding to a maximum delay of $4 \mathrm{~ns}$. The pump was directed onto the sample at close to normal incidence while the probe was incident at a larger angle. The reflected probe beam was directed into the same optical bridge detector used in the static measurements. The incident probe was $s$-polarized while the pump was passed through a quarter-wave plate followed by a linear polarizer so that its polarization could be rotated continuously while maintaining a constant intensity. The pump and probe were initially focused onto the surface of a laser beam profiler with lenses of 250 and $63 \mathrm{~mm}$ focal length respectively, mounted on three-axis translation stages. The pump and probe spots were observed to have $1 / e^{2}$ diameters of 98 and $48 \mu \mathrm{m}$ when focused at normal incidence. The laser beam profiler was also used to overlap the pump and probe spots before being removed so that the sample could be placed in the focal plane and between the pole pieces of an electromagnet. Two different electromagnets, ${ }^{54,55}$ designed for optimal optical access, were used to apply a magnetic field either perpendicular to (polar magnet) or in the plane of (longitudinal magnet) the sample. The polar (longitudinal) electromagnets could deliver maximum fields of about $\pm 11 \mathrm{kG}( \pm 4 \mathrm{kG})$ and allowed the angle of incidence of the probe to be set to $9^{\circ}\left(45^{\circ}\right)$. The overlap of pump and probe beams on the sample surface was monitored continuously with a CCD camera fitted with a macro lens. The pump was chopped at a frequency of $690 \mathrm{~Hz}$, and two lock-in amplifiers were used to extract the pump-induced modulation of the rotation and reflectivity outputs of the optical bridge. A further fine adjustment of the overlap of pump and probe spots could be performed by maximizing the output of the lock-in amplifiers at a fixed time delay.

All-optical pump-probe measurements have been used previously to study the precessional dynamics of optically opaque ferromagnetic thin films, multilayers, and nanostructures. ${ }^{49-51,56-59}$ The pump pulse is used to partially demagnetize the sample on sub-picosecond time scales. Consequently, dipolar fields, and in some cases effective fields associated with other interactions, are modified on similarly short time scales. If the total effective field is no longer collinear with the magnetization then the resulting torque will stimulate precessional motion. The instantaneous state of the magnetization is determined through a MOKE measurement made with the time-delayed probe pulse. The pump fluence should not be too large if the material parameters of the sample are to be treated as being uniform within the sample. The penetration of pump and probe pulses into the sample is characterized by the optical skin depth. ${ }^{60}$ Where the skin depth is smaller than the sample thickness, it should be remembered that the magnetization is most strongly excited at the upper surface of the sample and that the probe preferentially senses the response at the upper surface. It is often sufficient to 

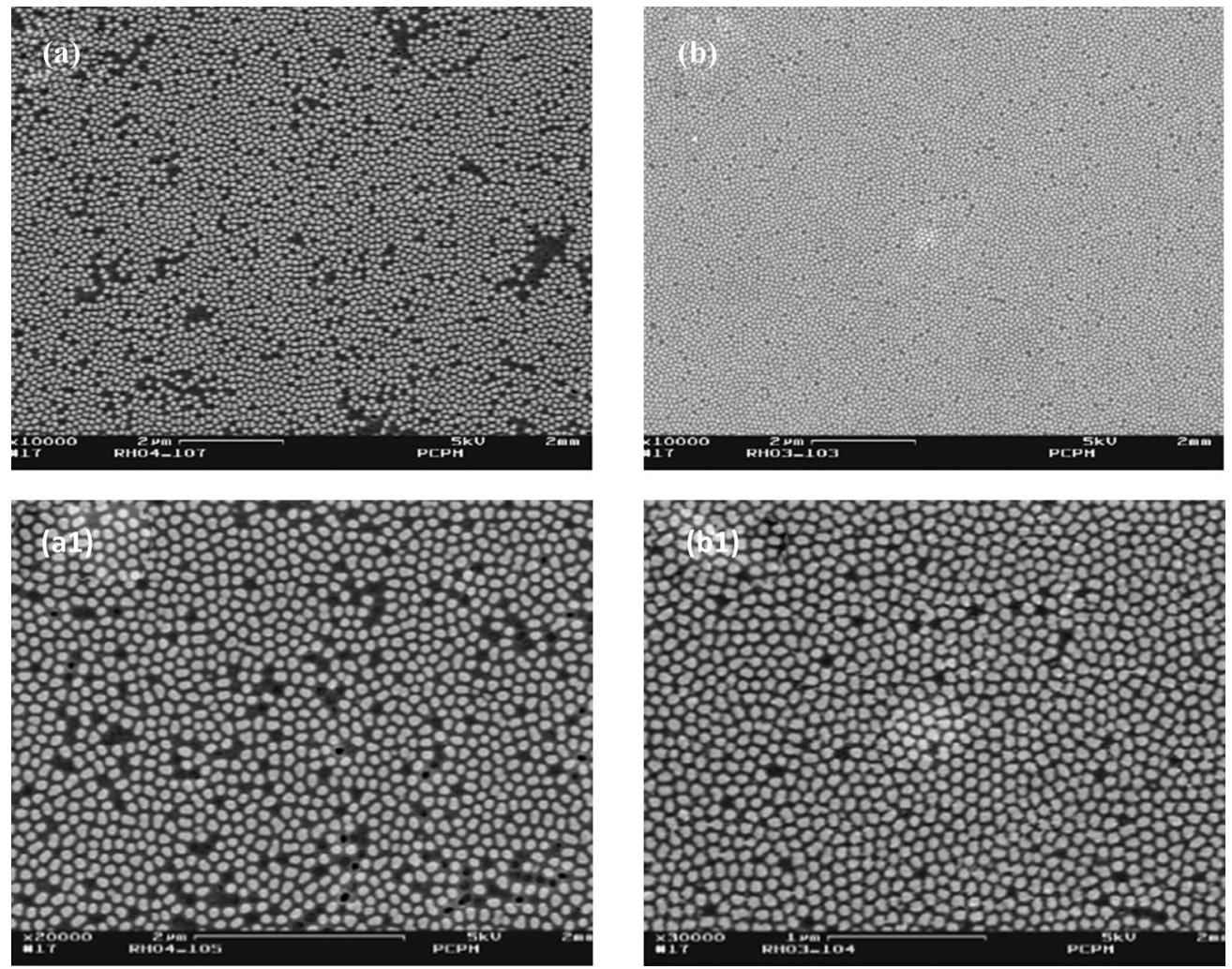

FIG. 1. SEM images of experimentally measured nanowire arrays (a) and (b) with 10000 magnification; (a1) the same array as (a) with 20000 magnification; (b1) the same array as (b) with 30000 magnification.

assume that the exchange interaction forces the motion of the magnetization to be uniform through the thickness of the sample. However, this will not be the case in the present study, since the magnetization becomes nonuniform at the end of each nanowire in order to minimize stray flux, and interpretation of the measured response requires this depth sensitivity to be taken into account.

The time-resolved Kerr rotation signal obtained from nanopatterned samples often contains oscillations associated with both magnetic and acoustic modes. The acoustic modes have frequency and phase that are independent of the applied magnetic field, while the phase of the magnetic modes changes by $180^{\circ}$ when the direction of the magnetization is reversed. The acoustic modes are not of interest in the present study and so the magnetic signal was isolated by considering the difference of signals acquired in magnetic fields of opposite polarity. However, extensive measurements were first performed to ensure that the time-resolved Kerr signals were not dependent upon the magnetic field history prior to the measurement. This conclusion is supported by the observation that the hysteresis loops are anhysteretic as is shown later.

Micromagnetic simulations were performed by using a unit cell of two neighboring nanowires and a macrogeometry approach $^{61}$ (or quasiperiodic boundary conditions, quasiPBCs) within the finite element micromagnetic code NMAG. ${ }^{48}$ The aim of applying PBCs in simulations is to reduce the effect of the finite size, i.e., by modifying the internal field in a finite array so that it can be treated as a part of an effectively infinite array. In NMAG, this is realized by creating a finite number of virtual copies of the simulated object (i.e., of the two wires in our case). The magnetization dynamics in each virtual copy is identical to that in the simulated object, but takes into account both static and dynamic dipolar fields produced by both the full ensemble of virtual copies and the simulated object. More specifically, simulations are performed for a pair of wires at the center of the array, shaded darker in Fig. 2, and the dipolar field inside these nanowires is calculated from the entire array generated by tessellating these nanowires with the translational lattice vectors $\mathbf{a}_{1}$ and $\mathbf{a}_{2}$. In the NMAG simulations reported here, the number of virtual copies was chosen so as

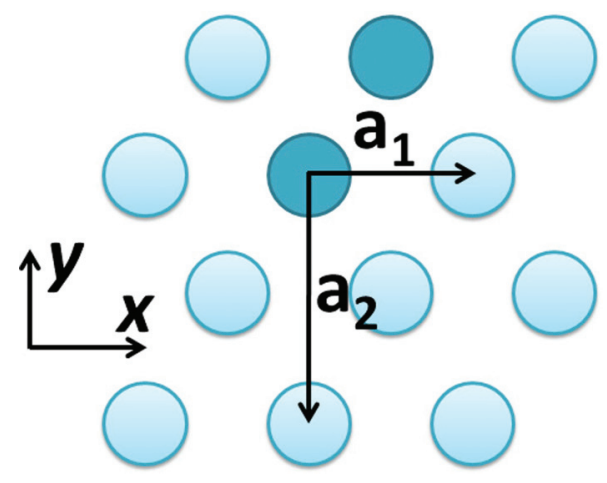

FIG. 2. (Color online) The geometry of the simulated array. NMAG simulations were performed for the two nanowires (shaded dark) as part of a quasi-infinite array created by tessellating virtual copies of the two nanowires with the primitive lattice vectors $\mathbf{a}_{1}$ and $\mathbf{a}_{2}$. The dimensions of the full array were $\sim 8 \times 8 \times 0.8 \mu \mathrm{m}$. 
to generate an array of size $\sim 8 \times 8 \times 0.8 \mu \mathrm{m}$. In the case of a MNWA with $d=85 \mathrm{~nm}$ and $p=0.66$ this corresponds to a $79 \times 90$ array, while for $p=0.4$ a $63 \times 74$ array is required. This yields an aspect ratio for the whole array of $\sim 1 / 10$. It has been shown ${ }^{62,63}$ that this aspect ratio guarantees a negligibly small influence of the borders on the central part of the system and also an insignificant difference of the dipolar field in the central two nanowires, which we simulated, to the dipolar field in these nanowires in an infinite array, which could be obtained by further decreasing the aspect ratio. Thus, the arrays studied here can be considered effectively infinite in the sense described above, i.e., "quasi-infinite." As we shall see, this simple idealized model can reproduce the main features of the experimentally measured arrays rather well.

Simulations were performed for magnetic parameters typical of Py, namely, saturation magnetization $M_{S}=800 \mathrm{G}$, exchange stiffness constant $A=1.1 \mu \mathrm{erg} / \mathrm{cm}$, and gyromagnetic ratio $\gamma=2 \pi \cdot 2.8 \mathrm{GHz} / \mathrm{kOe}$. A somewhat small value of the Gilbert damping constant $\alpha=0.005$ was used in the dynamic simulations so as to clearly reveal the structure of the eigenmodes and reduce their spectral width and to aid investigation of spin waves propagating along the nanowires. All simulations used a mesh with average dimensions of $5 \mathrm{~nm}$.

Static hysteresis loops were simulated, with the field applied both parallel and perpendicular to the length of the nanowires, first for $d=85 \mathrm{~nm}$ and edge-to-edge separations of $43.0 \mathrm{~nm}$ $(p=0.4)$ and $15 \mathrm{~nm}(p=0.66)$ and second for $d=110$ $\mathrm{nm}$ and edge-to-edge separations of $55.6 \mathrm{~nm}(p=0.4)$ and $40 \mathrm{~nm}(p=0.49)$. The length of the nanowires was $L=800$ $\mathrm{nm}$ in all cases. The magnetic field was first swept from 11.3 to $-11.3 \mathrm{kOe}$. The step size was 88.2 Oe from 11.3 to 2.3 $\mathrm{kOe}, 8.9$ Oe from 2.3 to $-2.3 \mathrm{kOe}$, and 88.2 Oe again from -2.3 to $-11.3 \mathrm{kOe}$. The field was then swept in the opposite direction from -11.3 to $11.3 \mathrm{kOe}$ using similar step sizes. The same sweep ranges were used for the fields applied both parallel and perpendicular to the length of the nanowires.

The following procedure was used to perform the dynamical simulations. ${ }^{64}$ First, the static magnetization configuration was calculated for a particular bias field value and used as the initial magnetization configuration for the dynamical simulations. One of three different stimuli were then applied: (i) a magnetic pulse with duration of $30 \mathrm{ps}$ and amplitude of 20 Oe applied to the full volume of the array; (ii) a pulse with the same duration and amplitude but applied to only the top $50 \mathrm{~nm}$ of the array; and (iii) a field applied to either the middle or top $10 \mathrm{~nm}$ of the array with temporal form described by the "sinc" function

$$
\mathbf{h}^{\prime}=h_{\max } \frac{\sin \left[2 \pi f_{0}\left(t-t_{0}\right)\right]}{2 \pi f_{0}\left(t-t_{0}\right)},
$$

with amplitude $h_{\max }=20$ Oe and cutoff frequency $f_{0}=$ $50 \mathrm{GHz}$. The parameter $t_{0}=10 / 2 f_{0}$ shifts the center of the "sinc" curve forward in time by five cycles of the cutoff frequency. The power spectrum of the pulse described by Eq. (1) has almost constant value for frequencies up to $f_{0}$ and then almost zero value above $f_{0}$. This ensures that all eigenmodes with frequencies $f<f_{0}$ are excited with approximately equal "strength." The stimulus (iii) was used only to improve the quality of the dispersion curves obtained for spin waves propagating along the nanowires, while all other results were obtained with stimuli (i) and (ii). Power spectra were obtained from a spatial average of the magnetization over either the whole volume or 50-nm-long sections at the top, middle, or bottom of the sample. The magnetization dynamics were recorded for $10.24 \mathrm{~ns}$ with the spatially resolved magnetization profile being saved after every 10 ps.

\section{STATIC PROPERTIES}

Hysteresis loops acquired from 1D arrays $^{39-41}$ and 2D arrays of high aspect ratio, ${ }^{35,37,38}$ with the magnetic field applied parallel to the axis of the nanowires ("parallel field geometry"), have finite coercive field and a nonvanishing remanence that depends upon the field history. The finite remanence is a consequence of the easy-axis shape anisotropy. The situation is different in arrays of low aspect ratio and, as will be shown, is the result of competition between the different demagnetizing fields associated with the shapes of the individual nanowires and of the array as a whole. The strength of the latter demagnetizing field, and consequently the reversal mechanism, strongly depends upon the packing density $(p)$. In Ref. 12, measurements made on arrays of Fe nanowires with $p=0.32(d=50 \mathrm{~nm}$ and edge-to-edge separation $35 \mathrm{~nm})$ yielded a coercive field of about $0.1 \mathrm{mT}$ when the field was applied along the length of the nanowires. In Refs. 19-21, zero coercive field was observed, irrespective of the direction of the applied magnetic field, for $\mathrm{Ni}$ and Py arrays with $p$ $\geqslant 0.35$. In Ref. 37, simulations of Py arrays with $p=0.4$ ( $d=10 \mathrm{~nm}$ and edge-to-edge separation of $5 \mathrm{~nm}$ ) yielded a coercive field of about $0.35 \mathrm{mT}$ when the magnetic field was applied along the length of the nanowires. This appears to be due to the extremely small nanowire diameter (only $10 \mathrm{~nm}$ ) assumed in the simulation. The diameter is comparable to the exchange length and so both the exchange interaction and the shape anisotropies influence the magnetization reversal. The exchange interaction favors uniform magnetization within each nanowire, which then behaves as a macrospin. It follows from Ref. 37 that the switching of these arrays occurs through the sudden coherent reversal of individual nanowires at different field values. It will be shown that the thicker nanowires considered in Refs. 19-21 reverse gradually through multidomain states. The effect of the nanowire diameter on the coercivity was also studied experimentally in arrays of $\mathrm{Fe}$ nanowires ${ }^{18}$ for diameters in the range $7-160 \mathrm{~nm}$ (the packing densities were not fixed and lie in the range $p=0.3-0.5)$. The coercivity was found to decrease sharply as the diameter of the nanowires increased. The same conclusions were reached in Ref. 65, where the authors considered magnetization reversal in arrays of Ni nanowires with different diameters.

Using the results of micromagnetic simulations that agree well with experiment, it will be shown that, for high packing densities $(p>0.4$ in the simulations), the easy-plane shape anisotropy associated with the array as a whole overcomes the easy-axis shape anisotropy of the individual nanowires and causes the coercivity and remanence to vanish, irrespective of the direction of the applied field.

Hysteresis loops acquired with the field applied parallel and perpendicular ("perpendicular field geometry") to the length of the nanowires are shown in Fig. 3. Figure 4 shows the simulated magnetization distribution in a pair of adjacent nanowires with $d=85 \mathrm{~nm}$ and with length of $800 \mathrm{~nm}$ that were 

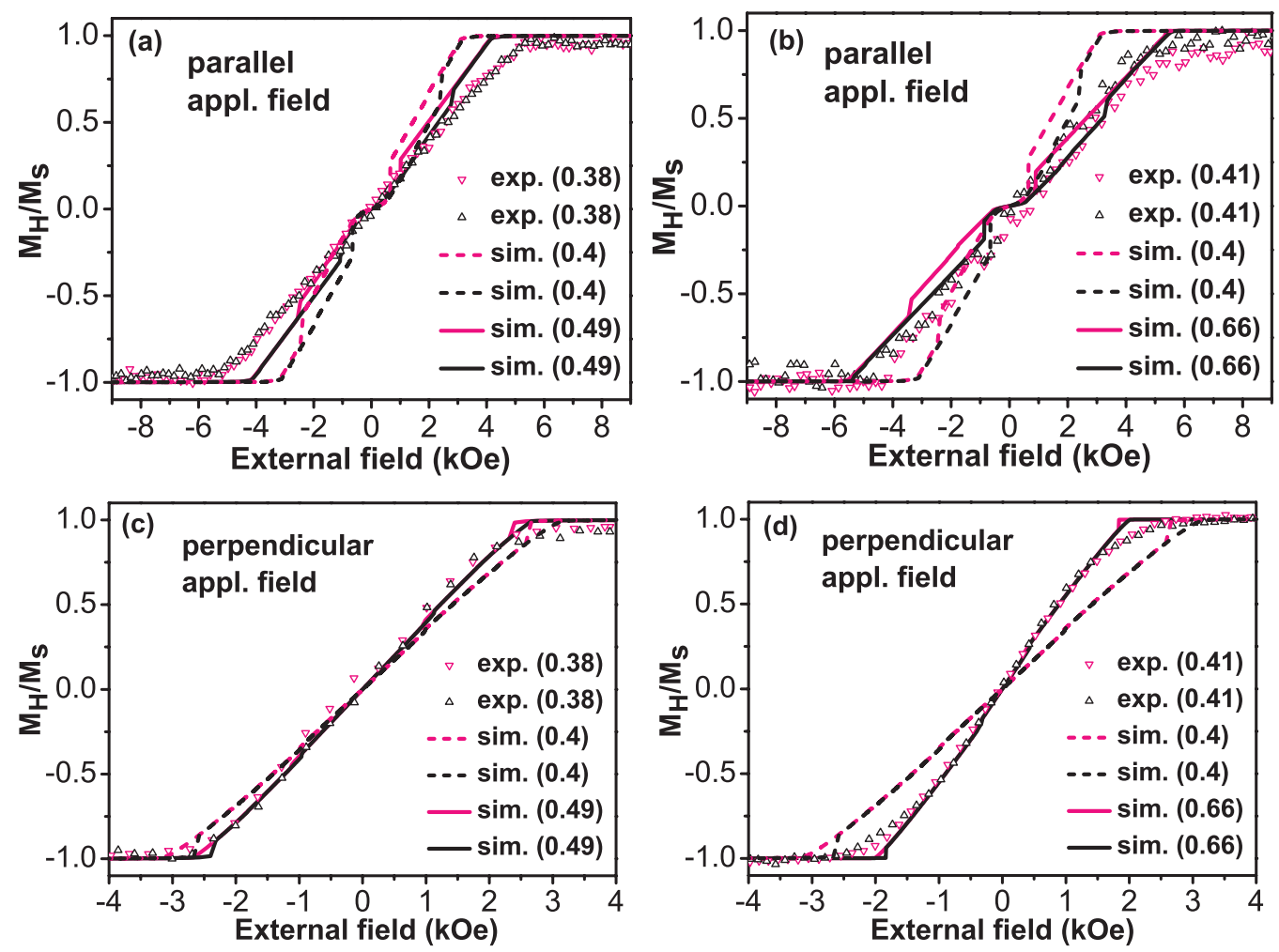

FIG. 3. (Color online) Hysteresis loops acquired with the field applied parallel and perpendicular to the length of the nanowires. Triangular symbols represent experimental results for two different samples with nominal $p=0.38$ and diameter $d=110$ in panels (a) and (c) and nominal $p=0.41$ and $d=85$ in panels (b) and (d). Dashed and solid lines show the results of simulations for the packing densities shown within the legend. Red curves correspond to field sweeps from positive to negative values while black curves represent the negative-to-positive case. The simulations reproduce the zero remanence and coercivity observed within the experiment.

repeated to form an array with $p=0.66$. The experimental and simulated hysteresis loops in Fig. 3 exhibit zero remanence and zero coercivity for both parallel and perpendicular field geometries. This behavior differs from that observed in arrays with low packing densities (for $p<0.35$ in Refs. 19-21), where zero remanence and coercive field were observed only in the perpendicular geometry. ${ }^{21,37}$ The qualitatively different behavior for arrays with high packing densities ( $p>0.4$ in the present study) occurs due to competition between the different shape anisotropies that have comparable strength in arrays of high packing density. For smaller packing density the easyplane shape anisotropy of the array decreases and the easy-axis shape anisotropy of the individual nanowires is dominant. As shown in Fig. 4, for large $p$ the competition between the shape anisotropies results in antiferromagnetic ordering, and hence zero coercivity and remanence, irrespective of the field history. This ground state is very robust. When a static field is applied, the magnetization tends to arrange itself so that there is no moment in directions orthogonal to the field. However, Fig. 4 shows that the magnetization reversal process is significantly different for the parallel and perpendicular geometries. In the former case the magnetization reversal occurs through multidomain states while in the latter case the magnetization gradually and uniformly rotates towards the direction of the applied field. Figure 3 shows that, unlike for the experimental case, the simulations produce minor loops. Their origin is discussed later.
The simulation procedure, by which a pair of nanowires is duplicated to define an array, leads to an idealized case of alternating rows magnetized in opposite directions, i.e., antiferromagnetic order, at zero applied field. In real arrays, the magnetic order might be regular with a different period or irregular as a result of structural disorder. This issue could be explored by including more than two nanowires in the unit cell. It was shown experimentally ${ }^{13,47}$ that in arrays with a pronounced easy-axis parallel to the length of the wires, the nanowires reverse in an irregular rather than periodic fashion in response to the applied field. However, these arrays showed a clear antiferromagnetic ground state ("checkerboard pattern") at zero applied field after the application of an ac demagnetizing field. In Ref. 47, a clear antiferromagnetic ground state was also observed in square MNWAs with nanowire aspect ratio $\sim 2$ and nominal packing density $p=$ 0.38. Defects and imperfections within real arrays (see Fig. 1) lead to local nonuniformities of the internal fields that may favor an aperiodic ground state. However, the success of the present simulations suggests that the ground state still has strong antiferromagnetic character.

Densely packed MNWAs with essentially zero remanence and coercivity are of particular interest because they have a robust remanent state with a reproducible microwave frequency response that is independent of field history. They therefore have the potential to replace ferrite materials in microwave devices of reduced size and energy consumption. ${ }^{6,9}$ 

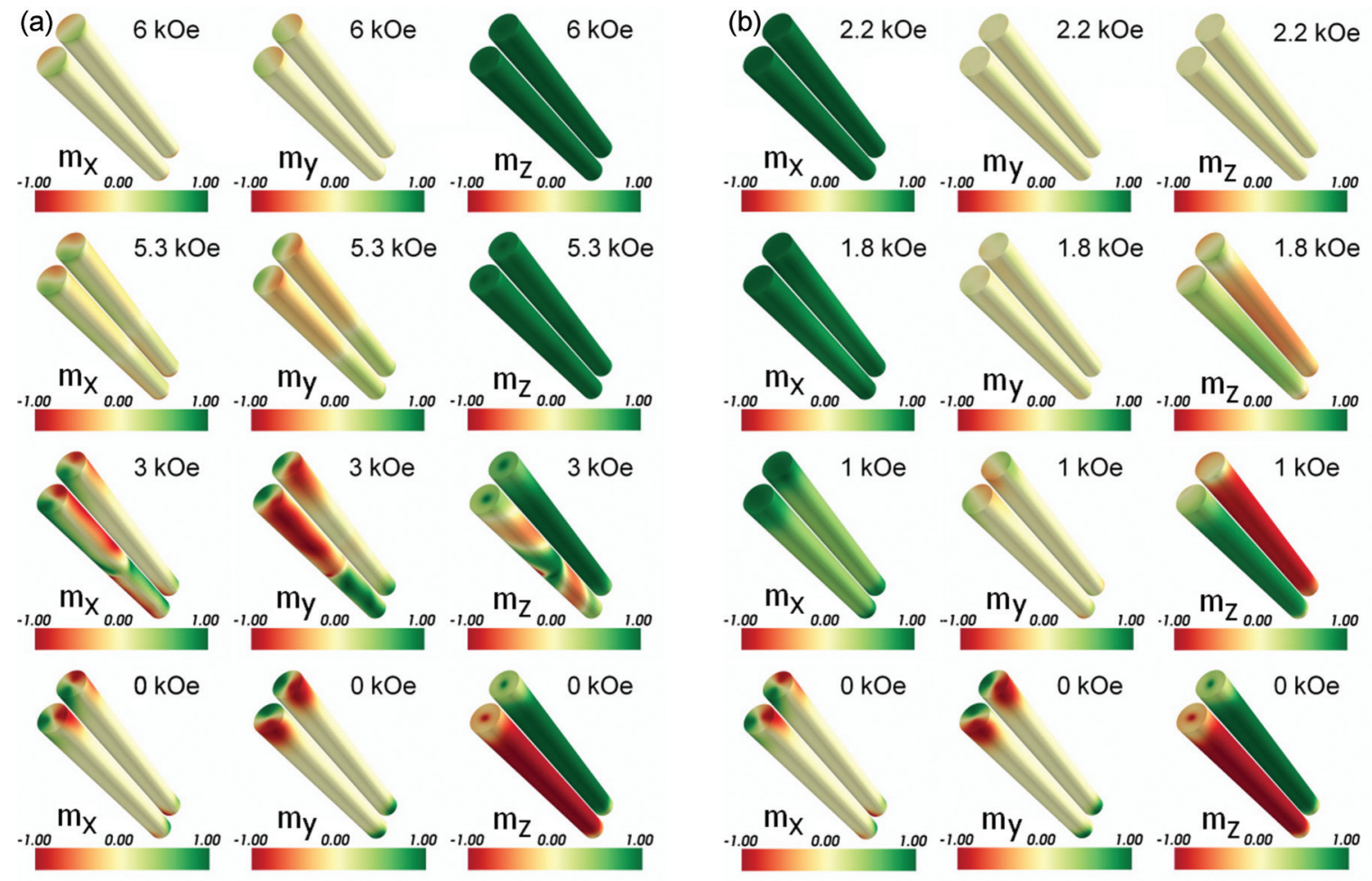

FIG. 4. (Color online) The magnetization distribution within a pair of adjacent nanowires from an array with $d=85 \mathrm{~nm}$ and $p=0.66$ for different values of the field applied (a) parallel and (b) perpendicular to the length of the nanowires. The color scale represents the value of each component of the normalized magnetization, $m_{x}, \mathrm{~m}_{y}, \mathrm{~m}_{z}$, from -1 (dark red) to 1 (dark green), displayed from left to right. The $z$ axis lies parallel to the length of the nanowire.

Such devices are expected to have a more stable response and do not require bulky bias magnets. The fact that the nanowire array generates zero stray field may also be an advantage for systems level integration.

In this paper, we consider the ground state and static properties of MNWAs with nanowire length one order of magnitude larger than the nanowire diameter, and with nanowire diameters in the range $85-110 \mathrm{~nm}$ that are significantly larger than the exchange length. A comprehensive study of the influence of the length of the nanowire (for aspect ratios in the range 0-3) and the nanowire diameter (considered in units of exchange length in the range 0-12) on the ground state of the single nanowire and its static properties can be found in Ref. 13. A diagram was presented (Fig. 6 in Ref. 13) that showed the different single nanowire ground states obtained for different values of these two nanowire parameters. The diagram was used to explain the different types of experimental hysteresis loops obtained from $\mathrm{Ni}, \mathrm{Co}$, $\mathrm{CoNi}$, and CoP nanowire/nanoparticle (depending on particle aspect ratio) arrays. The present study could, in principle, be extended by generating similar diagrams for two nearestneighbor nanowires/nanoparticles within arrays of different packing density. The possible magnetization distributions within a single nanowire from a pair of nanowires within the array are expected to be essentially the same as shown for an isolated nanowire in Ref. 13. However, the parameter values at which transitions occur between different types of magnetization distribution will be different, within a single nanowire from a pair of nanowires within the array, as compared to an isolated nanowire. Such diagrams obtained for a pair of nanowires within an array may offer a better explanation of the features of the hysteresis loops obtained from measurements of different arrays.

To explore the origin of the minor hysteresis loops seen in the simulations and their dependence upon the nanowire diameter, simulations are shown in Fig. 5 for two arrays with packing density $p=0.4$ and nanowire diameters of $d=85$ and $110 \mathrm{~nm}$. The minor loops seen in the simulations are the result of the finite nanowire radius, the influence of the exchange interaction, and pinning at the nanowire boundaries. When the nanowire radius is increased from 85 to $110 \mathrm{~nm}$, the minor loops become smaller for the case that the field is applied parallel to the length of the nanowires. If the diameter of the nanowires is instead decreased so as to become comparable to the exchange length, then the two minor loops increase in area and merge, leading to nonzero coercivity and remanence (see Ref. 37 for hysteresis loops from nanowire arrays with $10 \mathrm{~nm}$ diameter). The simulations also show that the saturation field increases from 3.1 to $3.3 \mathrm{kOe}$ in the parallel field geometry when the nanowire diameter is increased from 85 to $110 \mathrm{~nm}$. For the case where the field is applied perpendicular to the length of the nanowires, the area of the minor loop that occurs close to the saturation field becomes slightly larger as the nanowire radius increases. This minor loop is the result of pinning of the magnetization at the boundaries of the nanowire, and the sharp transition 

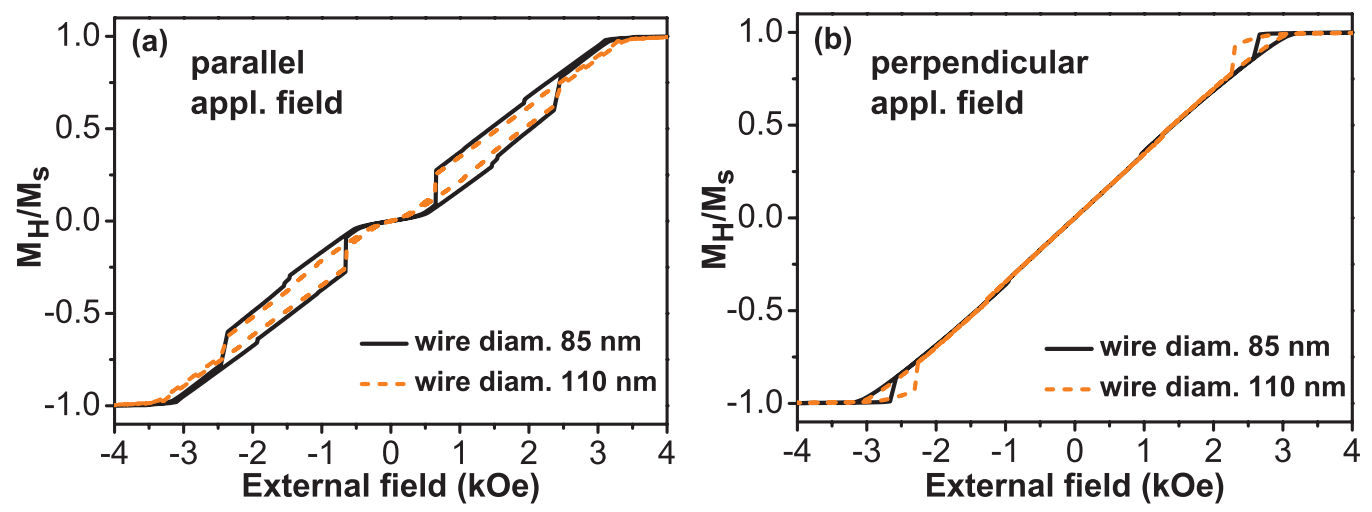

FIG. 5. (Color online) Simulated hysteresis loops for nanowire arrays with packing density $p=0.4$ and $d=85 \mathrm{~nm}$ (black solid lines) and $110 \mathrm{~nm}$ (dashed orange lines) for both parallel and perpendicular applied magnetic fields. The field was swept from both the positive to negative direction and vice versa.

corresponds to a small angle switching of the magnetization direction. The saturation field is the same for both nanowire diameters in the perpendicular field geometry.

The minor loops seen in the simulations are not observed in the experiment. The loops might not be seen in the experiment due to the finite resolution of the measurement technique (drawing a line through the triangles in Fig. 3 leads to wavy curves that cross each other many times as the field is swept up and down) or due to inhomogeneous broadening within the array that leads to a distribution of minor loop transition fields. Comparing Figs. 3 and 5, it can be seen that variation of the nanowire diameter in the range considered here does not influence the static properties of the arrays significantly compared to the effect of changing the packing density. Therefore, it is principally the packing density that determines the strength of the easy-plane shape anisotropy that is of primary importance in determining the static properties of the arrays considered in the present study.

Table I presents the experimental and simulated values of the saturation field for both parallel and perpendicular geometries. The simulations show that the saturation field increases with packing density for the parallel field geometry but decreases with packing density for the perpendicular field geometry, which can be understood as follows. In the limiting case of a ferromagnetic film $(p=1)$ the saturation field should be zero when the field is applied in the plane of the film (perpendicular field geometry) and have value equal to $4 \pi M_{S} \sim 10 \mathrm{kOe}$ (for Py) when the field is applied

TABLE I. Simulated and experimental values of saturation field for parallel and perpendicular field geometries and different packing densities.

\begin{tabular}{|c|c|c|c|c|c|}
\hline & $\begin{array}{c}p=0.38 \\
(\mathrm{kOe})\end{array}$ & $\begin{array}{c}p=0.4 \\
(\mathrm{kOe})\end{array}$ & $\begin{array}{c}p=0.41 \\
(\mathrm{kOe})\end{array}$ & $\begin{array}{c}p=0.49 \\
(\mathrm{kOe})\end{array}$ & $\begin{array}{c}p=0.66 \\
(\mathrm{kOe})\end{array}$ \\
\hline & \multicolumn{5}{|c|}{ Parallel applied field } \\
\hline Experiment & \multirow[t]{2}{*}{5.3} & \multirow{2}{*}{\multicolumn{2}{|c|}{4.7}} & & \\
\hline \multirow[t]{2}{*}{ Simulation } & & & & 4.2 & 5.5 \\
\hline & \multicolumn{5}{|c|}{ Perpendicular applied field } \\
\hline Experiment & 3 & & 2.4 & & \\
\hline Simulation & & 3.1 & & 2.6 & 2 \\
\hline
\end{tabular}

perpendicular to the plane of the film (parallel field geometry). Table I shows that in the experiments the array with slightly larger packing density has a saturation field that is smaller by $0.6 \mathrm{kOe}$ for both the parallel and perpendicular geometries. This decrease of the parallel geometry saturation field values is unexpected. Indeed, simulations show that the difference in nanowire diameter for the two experimental arrays may account for only one-third of this decrease of the parallel geometry saturation field value. The observed difference may be the result of disorder within the arrays (see Fig. 1) or some difference in the saturation magnetization in different samples resulting from the fabrication process.

The simulated saturation value for $p=0.4$ is close to the experimental value for $p=0.38$ in the perpendicular geometry. However, in the parallel geometry, the simulated saturation field for $p=0.4$ is smaller by $1.4-2 \mathrm{kOe}$ than the experimental values for the arrays with $p=0.38$ and 0.41 . Again the discrepancy may be the result of structural disorder, which produces variations in the local internal field that effectively pin the magnetization, preventing it from reorienting towards the direction of the applied field. As one can see, the simulated saturation values for larger values of $p$, which correspond to the minimum edge-to-edge separations observed within the arrays (i.e., if disorder was eliminated), are in better agreement with the experimental values for the parallel geometry but still in good agreement for the perpendicular geometry.

Both experiment and simulation exhibit a robust ground state with zero net remanent magnetization, irrespective of the field history. Individual nanowires are almost uniformly magnetized along their length, but within the array there are equal numbers of nanowires with magnetization pointing up and down relative to the substrate. The simulations considered the case of periodic antiferromagnetic order but this may not be necessary. The robust ground state may be very useful in microwave electronics where the same microwave absorption frequency can be reliably achieved at remanence.

\section{DYNAMIC PROPERTIES}

The experimental and simulated excitation spectra are now presented and compared for both parallel and perpendicular field geometries. TRMOKE measurements were made on 

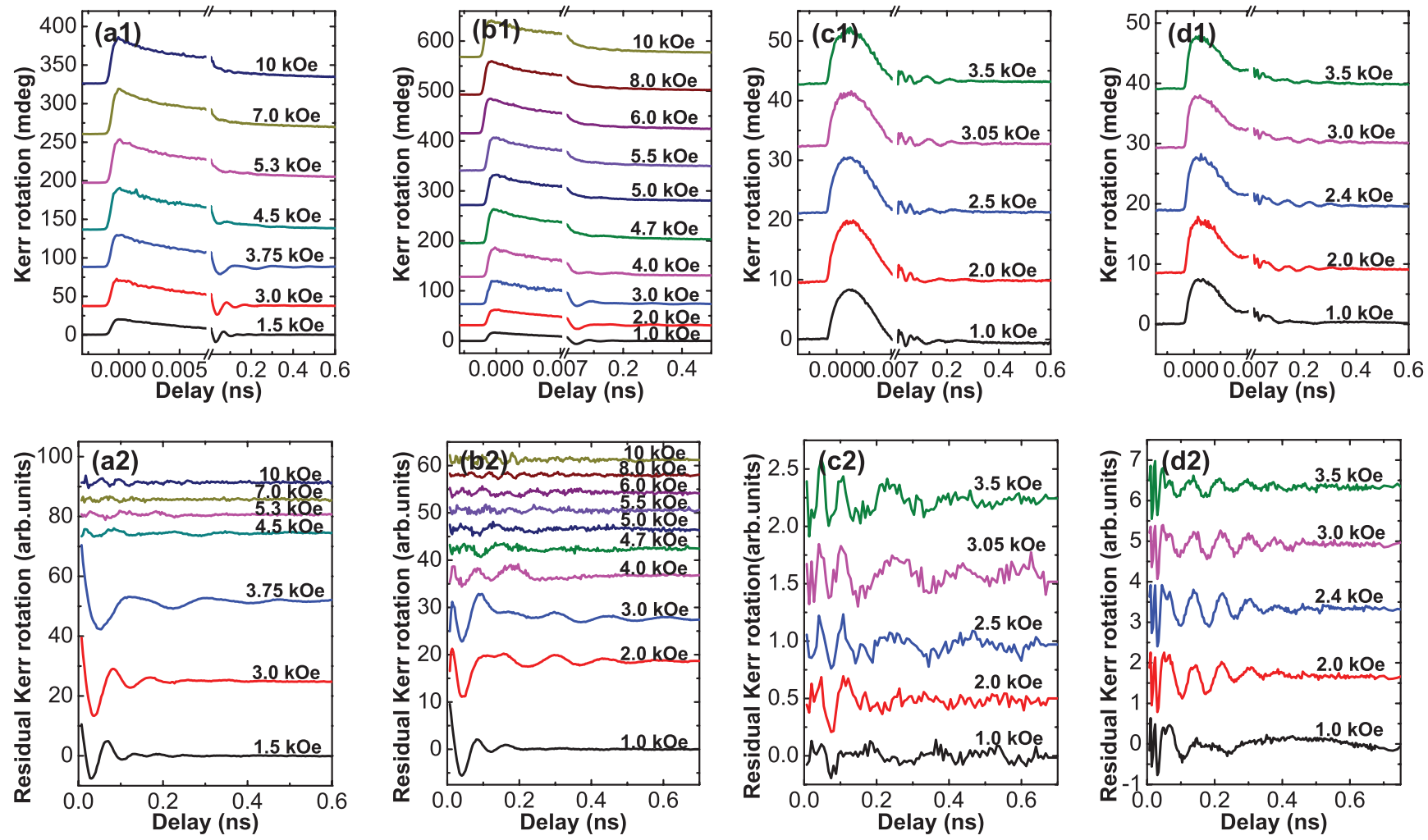

FIG. 6. (Color online) Transient MOKE response of MNWA before (a1)-(d1) and after (a2)-(d2) a multiexponential background has been subtracted from each scan in the parallel field geometry for (a1),(a2) $p=0.38$ and (b1),(b2) $p=0.41$ and in the perpendicular field geometry for (c1),(c2) $p=0.38$ and (d1),(d2) $p=0.41$.

both samples while simulations were performed for arrays of wires with $d=85 \mathrm{~nm}$ and $p=0.4$ and 0.66 and also for a single nanowire. It will be shown that competition between easy-axis and easy-plane anisotropies of comparable strength in densely packed arrays leads to dynamical properties that are significantly different from those of both a single wire and a continuous magnetic film. The nanowires are strongly coupled by dipolar fields that lead to collective excitations and a band structure associated with the components of the wave vector $\mathbf{k}$ that lie in the plane of the array (the $x y$ plane in the present case). Each band originates from a mode supported by a single nanowire. The large size of the focused spots within the TRMOKE measurements favors collective excitations with values of the in-plane wave vector very close to the center of the Brillouin zone but, as is shown later, the antiferromagnetic ground state leads to collective excitations with nonzero wave vector in the plane of the array. These collective excitations can, of course, possess any value of the wave vector component $k_{z}$ that lies parallel to the length of the nanowires.

Figure 6 shows the results of TRMOKE measurements made in the parallel and perpendicular geometries on the nanowire arrays with $p=0.38$ and 0.41 . The transient Kerr rotation signal rises within a few hundred fs due to the ultrafast demagnetization induced by the pump pulse. It then exhibits a multiexponential decay on which oscillations associated with magnetization precession are superimposed. Panels (a1)-(d1) represent the signal before and panels (a2)-(d2) after the nonoscillatory background was fitted and then subtracted. The background will be described elsewhere since it is not relevant to the discussion of the precessional excitation spectrum of the sample.

\section{A. Parallel field geometry}

Figures 7(a) and 7(b) show the experimental spectra obtained from arrays with nominal $d=110$ and $85 \mathrm{~nm}$, respectively, and nominal $p=0.38$ and 0.41 , respectively, with the field applied parallel to the length of the nanowires and therefore perpendicular to the plane of the array ("parallel field geometry"). For both arrays, the experimental spectra contain a number of broad peaks. While the general trend of the variation of the resonant peaks with applied field is similar for the two samples, there are also significant differences despite their similar packing densities. For $p=0.38$, the spectra exhibit a prominent peak with frequency that decreases as the applied field is increased from 0 to $3.75 \mathrm{kOe}$ (a value smaller than the saturation field of $5.3 \mathrm{kOe}$ ) and then increases as the field is increased further. For $p=0.41$, a prominent peak is observed only for the two lowest field values. From 3 to $5 \mathrm{kOe}$ (the saturation field is $4.7 \mathrm{kOe}$ ) the spectrum exhibits multiple peaks. From 5 kOe upwards there is a prominent peak with frequency that increases with field. The frequency of the prominent peak is larger for the sample with $p=0.38$ at small applied fields and for the sample with $p=0.41$ above saturation. The latter observation can be explained by the fact that the sample with $p=0.41$ has smaller saturation field and hence larger internal field for a particular field value above saturation. 

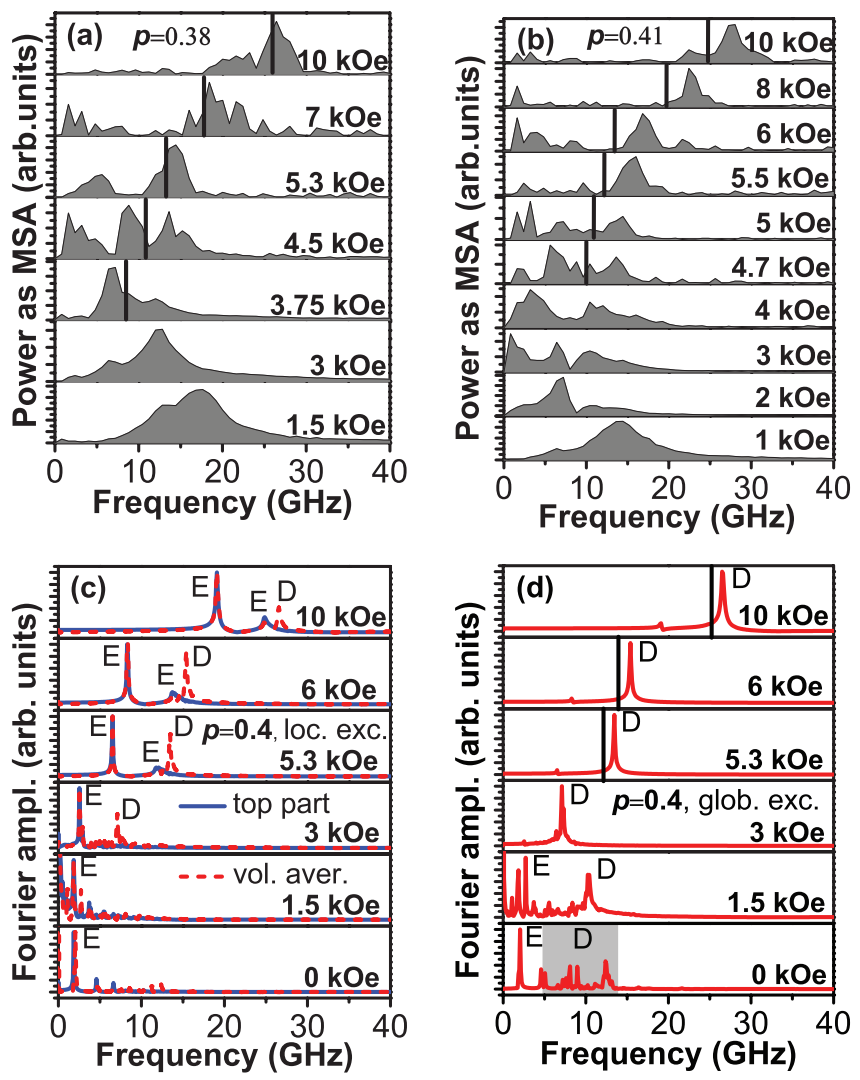

FIG. 7. (Color online) (a),(b) Power spectra obtained from TRMOKE measurements of arrays with $p=0.38$ and 0.41 , respectively, in the parallel field geometry. (c) Simulated spectra for an array with $p=0.4$, excited by a magnetic field pulse applied to the top $50 \mathrm{~nm}$ of the array, calculated from the response of the top $50 \mathrm{~nm}$ (solid blue line) and space averaged magnetization (dashed red line) of the array. E and D indicate end and delocalized modes, respectively. (d) Simulated spectra for an array with $p=0.4$, excited by a uniform pulsed field, calculated from the response of the space averaged magnetization of the array. Black solid vertical lines in panels (a), (b), and (d) indicate resonant frequencies calculated from Eq. (2) for the same $p$ values. All spectra are plotted on a linear scale.

Since the skin depth of bulk Py is the order of $20 \mathrm{~nm}$ at optical frequencies, it is reasonable to expect a somewhat larger value for a Py MNWA embedded within an alumina matrix. It was shown in Refs. 16-18 that Fe, Co, and CoPt MNWAs that are a few microns in length are sufficiently transparent for magneto-optical measurements to be performed in a transmission geometry. In light of these observations, we adopted a simple approach in performing dynamic micromagnetic simulations that could be compared to the results of TRMOKE measurements. First, the action of the pump was represented by a pulsed magnetic field applied to only the top $50 \mathrm{~nm}$ of the array. Then the response of the same top $50 \mathrm{~nm}$ was spatially averaged and plotted for comparison with experiment. Second, the whole sample was excited and then sensed in additional simulations to provide a fuller understanding and comparison with the experimental data available.

Figure 7(c) shows the simulated spectra for the top $50 \mathrm{~nm}$ (solid blue line) and the spatially averaged magnetization from the full volume (dashed red line) of an array with $d=85 \mathrm{~nm}$ and $p=0.4$ in response to a magnetic field pulse applied to the top $50 \mathrm{~nm}$ of the array. Analysis of the associated spatial mode profiles (not shown) showed that some of the peaks in the spectra are associated with end modes (labeled E) that are localized in wells of reduced internal field near the top surface of the array. Their frequencies are significantly smaller than those observed within the experiment. The spectra for the spatially averaged magnetization from the full thickness of the array exhibit additional strong peaks that result from delocalized modes (labeled D) and have higher frequencies that agree well with the experimental values. The results of simulations performed by exciting and sensing the full thickness of the array are shown in Fig. 7(d). The simulated spectra contain both $\mathrm{E}$ and $\mathrm{D}$ modes and are in better qualitative and quantitative correspondence with experiment. The experimental frequencies are consistently larger than those of the simulated $\mathrm{E}$ modes and quite similar to the frequencies observed within microwave ferromagnetic resonance measurements of densely packed arrays. ${ }^{20}$ This may be because the optical pump and probe pulses penetrate further than $50 \mathrm{~nm}$ into the array, perhaps due to waveguiding within the alumina, and are, in fact, able to access the middle section of the nanowires. This conclusion is supported by previous studies of how the magneto-optical properties of the array depend upon the length of the nanowires. ${ }^{16-18}$ Alternatively polishing the upper surface of the MNWA may lead to inhomogeneous broadening of the E modes so that the D modes appear more prominent in the experimental spectra.

Analysis of mode profiles, from simulations in which the full volume of the array was excited and sensed, show that a uniform mode is excited for fields above the saturation value. It is possible to write a simple phenomenological equation for the frequency of this fundamental mode,

$$
f=\gamma[\mathrm{H}+2 \pi(1-3 p) \mathrm{M}],
$$

by linking the limiting cases of a single wire magnetized along its length and a ferromagnetic film magnetized normal to its plane. ${ }^{20}$ The second term represents the shape anisotropy. Equation (2) gives the exact result for the frequency of the fundamental uniform mode of the single wire when $p=0$ and for the ferromagnetic film when $p=1$. The resonant frequencies predicted by Eq. (2) for the relevant packing densities are shown as black vertical lines in Figs. 7(a), 7(b), and 7(d). The experimental frequencies recorded above (and slightly below) saturation and the simulated frequencies obtained by exciting and sensing the full volume of the array are both well described by this simple phenomenological equation. This equation also describes the resonant frequencies of nanowire arrays obtained in FMR measurements. ${ }^{19,20}$

Comparing Figs. 7(a) and 7(d), the frequencies of the D modes observed in experiment and simulation decrease to roughly the same minimum value at similar values of the applied field, although the frequency decrease is more dramatic in the experiment. However, in the simulations the frequency decreases until the saturation field $(3.3 \mathrm{kOe})$ is reached, while in the experiment the frequency decreases up to a field value of $3.75 \mathrm{kOe}$ that is significantly lower than the saturation value $(5.3 \mathrm{kOe})$. After reaching the minimum, the frequencies increase with increasing applied field, exhibiting similar values and the same linear dependence on the applied field in both 

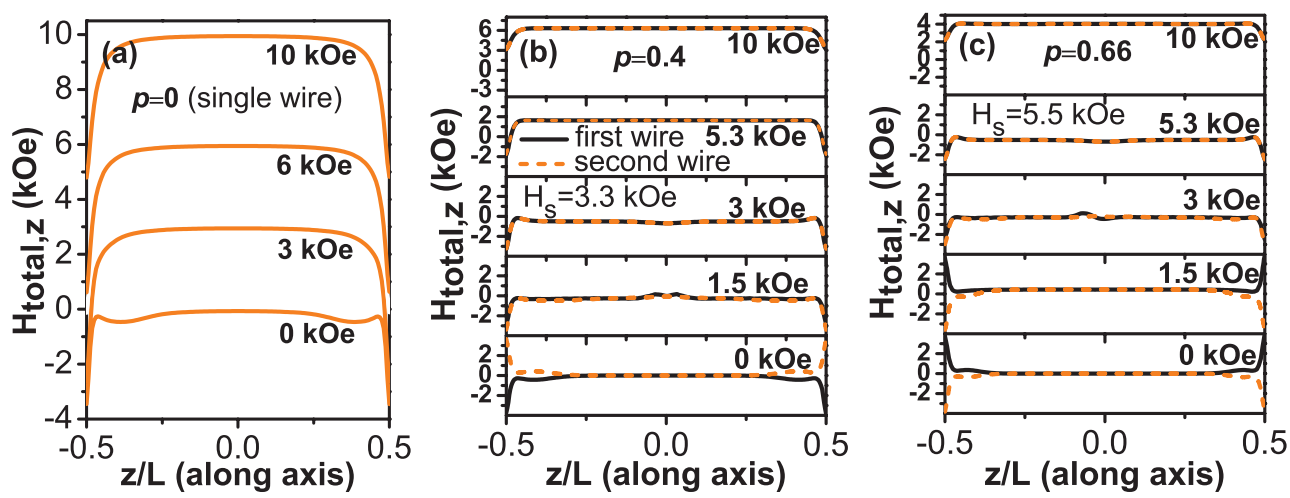

FIG. 8. (Color online) The component of the static internal field (sum of applied and demagnetizing fields) along the nanowire axis as a function of position on the axis in two neighboring nanowires ( $p=0$ corresponds to a single nanowire) with antiparallel magnetization at remanence in arrays with $d=85 \mathrm{~nm}$ and $p$ values of (a) 0 , (b) 0.4 , and (c) 0.66 . The value of the saturation field $H_{s}$ is indicated in panels (b) and (c).

experiment and simulation. The simulated and experimental frequencies of the D modes at $10 \mathrm{kOe}$ applied field have similar values of about $26.5 \mathrm{GHz}$ despite a significant difference in the saturation fields in experiment and simulation.

Figure 8 shows the component of the static internal field (the sum of the applied and demagnetizing fields) parallel to the nanowire axis plotted as a function of the position on the axis in two neighboring nanowires with antiparallel magnetization at remanence. Unlike a single wire, where increased applied field leads to an immediate increase of the mean internal field, the mean internal field in the arrays (apart from small regions at the ends of the nanowires) remains close to zero up until the MNWAs begin to saturate. This is because the demagnetizing field from the top and bottom surfaces of the array as a whole compensates the applied field.

As seen above, the experimental frequencies start to increase from a field value less than the saturation value, so unlike in the simulation, the mean internal field in the real array may begin to increase from a field value smaller than the saturation value, due to its irregular structure.

To understand why the measured and simulated mode frequencies initially decrease as the field is increased from remanence, it is necessary to consider the spin waves that propagate along the nanowires, their dispersion, and the mode profiles of collective excitations within the plane of the array. The case of a single nanowire is considered first. Figure 9(a) shows the simulated mode spectrum of a single nanowire ( $p=0$ ) with $d=85 \mathrm{~nm}$. The full length of the nanowire was excited and sensed. Analysis of the mode profiles shows that both $\mathrm{E}$ and fundamental $\mathrm{D}$ modes are excited by the uniform field. Although the E modes are localized in small end regions of the nanowire, they appear in the spectrum with comparable amplitude to the fundamental D modes. Not all E modes are visible within the spectrum. A fuller understanding of the $\mathrm{E}$ modes requires further analysis of the response of only the end region of the nanowire.

From Fig. 9(a), an increased applied field leads to an immediate increase of the resonant frequencies of both the $\mathrm{E}$ and D modes. Figures 9(b) and 9(c) show the lower branches of the dispersion relation for excitations propagating along the nanowire with $d=85 \mathrm{~nm}$ for applied fields of 0 and $6 \mathrm{kOe}$, respectively. The dispersion curves have backward-volumelike character (the wave vector is parallel to the magnetization and the group velocity is negative for small values of $k_{z}$ ) and the frequencies simply increase with applied field while the mode character is preserved. The flat branches within Figs. 9(b) and 9(c) correspond to localized E modes.

Figure 9(d) shows simulated spectra for an array with $d=$ $85 \mathrm{~nm}$ and $p=0.66$ that is excited and sensed through its full volume. Figures $9(\mathrm{e})-9(\mathrm{~g})$ show dispersion curves $^{66}$ for spin waves propagating along the nanowires within the array for applied fields of 0,6 , and $10 \mathrm{kOe}$, respectively. Comparison of the spectra with the dispersion curves and analysis of the mode profiles reveal that the first dominant peak at $0 \mathrm{kOe}$ corresponds to an E mode localized at the top and bottom surfaces of the array [see Figs. 9(d)]. The dominant peaks within the gray region, which have been labeled, are $\mathrm{D}$ modes that belong to a continuous spectrum. The corresponding points on the dispersion curves within Fig. 9(e) have also been labeled. Within this figure there are two branches of different character that occur due to competition between the different shape anisotropies. The lower parabolic branch, which has positive group velocity, is the result of the easy-plane anisotropy associated with the shape of the array as a whole. As the value of $p$ increases, the bottom of the parabola moves towards 0 GHz. The upper branch has backward-volume-like character. In the limiting case of a single nanowire the frequencies within this branch increase until at $k_{z}=0$ the frequency reaches that of the uniform ferromagnetic resonance of a long cylinder, which is about $14 \mathrm{GHz}$ in the present case. The two branches converge and become degenerate above a particular value of $k_{z}$. The three dominant peaks 1, 2, and 3 within Fig. 9(d) correspond to the bottom of the lower (parabolic) dispersion branch, the points of convergence, and the top of the upper branch at $k_{z}=0$, respectively. The convergence points correspond to a wavelength $\sim 90 \mathrm{~nm}$. This is the wavelength below which the exchange interaction dominates and above which dipolar interactions dominate.

The lower frequency peaks with large amplitude at fields of 1.5 and $3 \mathrm{kOe}$ within Fig. 9(d) correspond to end/edge modes. These modes are localized on both the top and the bottom surfaces, and also on the cylindrical boundaries of 

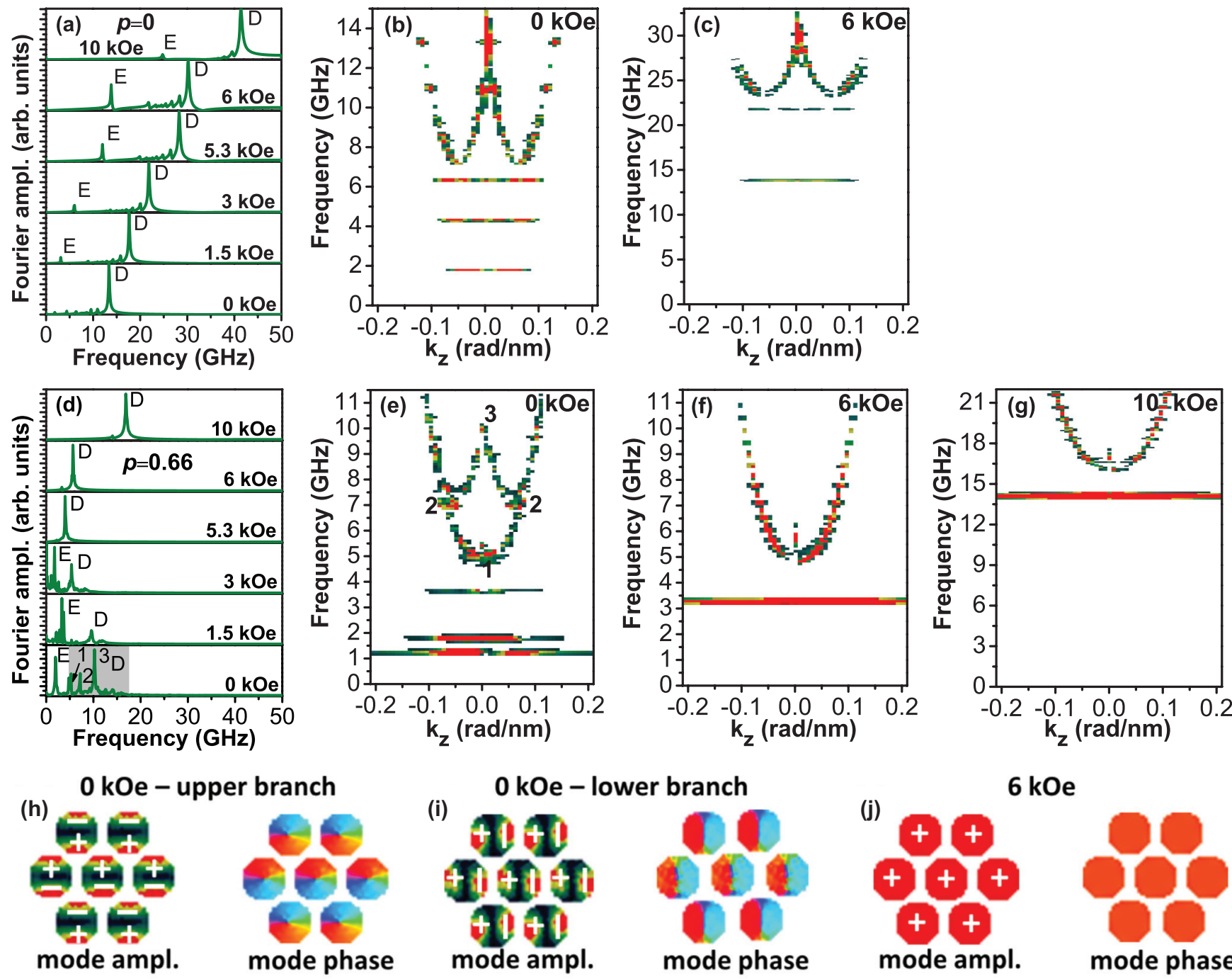

$6 \mathrm{kOe}$
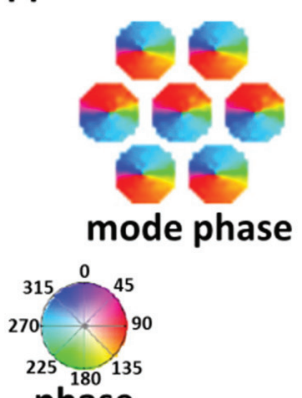

ampl. phase

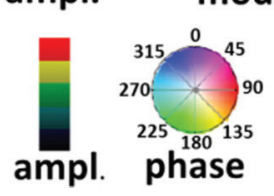

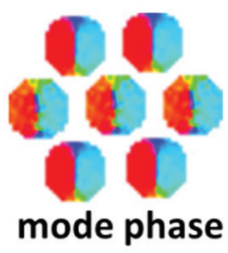

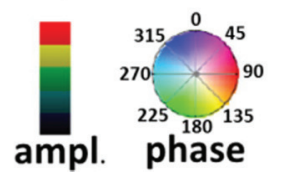

FIG. 9. (Color online) (a) Simulated spectra of a single nanowire $(p=0)$, with $d=85 \mathrm{~nm}$, excited and sensed along its full length. E and D denote end and delocalized modes. (b),(c) Dispersion curves for spin waves propagating along the nanowire for applied fields of 0 and $6 \mathrm{kOe}$, respectively. (d) Simulated spectra of a nanowire array with $d=85 \mathrm{~nm}$ and $p=0.66$ excited and sensed through its full volume. At $0 \mathrm{kOe}$, D modes are labeled in correspondence with the points in (e). (e),(f), (g) Dispersion curves for excitations propagating along the nanowires within the array for fields of 0,6, and $10 \mathrm{kOe}$, respectively. (h),(i),(j) Mode profiles (amplitude, left; and phase, right) within the plane of the array for $k_{z}=0$ for the upper and lower branches of the dispersion curve at 0 and $6 \mathrm{kOe}$, respectively.

the nanowires in demagnetizing wells created by the static magnetization. The demagnetizing wells on the cylindrical boundaries of the nanowires are created in nanowires that are undergoing quasistatic magnetization reversal as the nonuniform magnetization acquires components orthogonal to the cylindrical boundaries [see Fig. 4(a)]. A uniform pulsed field, applied to the full volume of the array, excites these modes with larger amplitude than the D modes. The higher frequency peaks of large amplitude at 1.5 and $3 \mathrm{kOe}$ correspond to D modes. The single peaks of large amplitude at 5.3,6, and $10 \mathrm{kOe}$ (slightly below and above saturation) are again the fundamental D modes.
Figures 9(h)-9(j) show the mode profiles ${ }^{66}$ (mode amplitude-left and phase-right) in the plane of the array for the lower and upper branches of the dispersion relation in Fig. 9(e) and the dispersion curve in Fig. 9(f), respectively. The mode profiles for the array have been generated by duplicating the mode profile of the unit cell at every lattice point. The two branches within Fig. 9(e) correspond to different mode profiles within the unit cell and hence within the plane of the array. These collective modes originate from antisymmetric modes of an individual wire where opposite sides of the cylindrical surface oscillate out of phase. The lower branch corresponds to the mode for which the facing edges of nearest-neighbor 

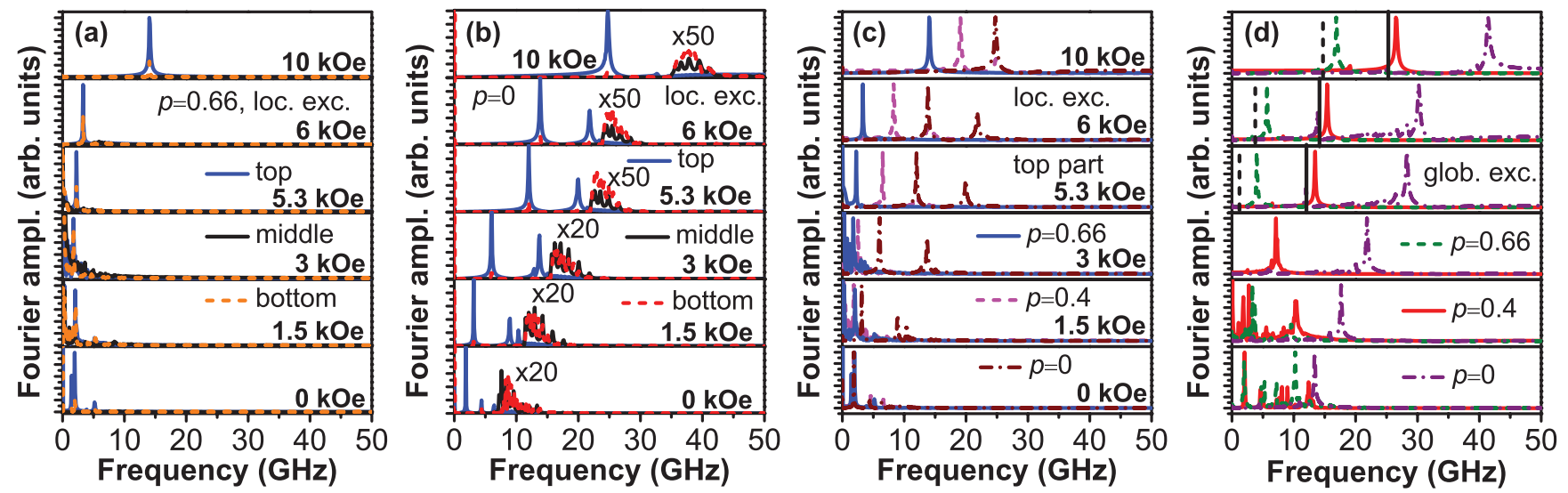

FIG. 10. (Color online) (a) Simulated spectra of an array of nanowires with $d=85 \mathrm{~nm}$ and $p=0.66$ in response to a pulse applied to only the top $50 \mathrm{~nm}$ of the array. The blue and black solid lines and red dashed line correspond to the responses of 50-nm-long regions at the top, middle, and bottom of the array, respectively. (b) Simulated spectra of a single wire with $d=85 \mathrm{~nm}$ in response to a pulse applied to only the top $50 \mathrm{~nm}$. The line styles have the same meaning as in (a). The response of the middle part is magnified by a factor of 10 , and that of the bottom part is magnified by a factor indicated separately for each panel. (c) Simulated spectra from the top $50 \mathrm{~nm}$ of an array of nanowires with $d=85 \mathrm{~nm}$ and $p=0.66$ (blue solid line), 0.4 (dashed magenta line), and 0, i.e., a single wire (dash-dotted brown line) in response to a magnetic pulse applied to the top $50 \mathrm{~nm}$ of the array. (d) Simulated spectra from the full volume of an array with $d=85 \mathrm{~nm}$ and $p=0.66$ (dashed green line), 0.4 (solid red line), and 0, i.e., a single wire (dashed dotted purple line) in response to a pulse applied to the full volume of the array. The applied field values are the same as stated in panels $(\mathrm{a}-\mathrm{c})$. Black solid and dashed vertical lines denote frequencies calculated from Eq. (2) with $p=0.4$ and 0.66 , respectively.

nanowires oscillate out of phase, leading to attenuation of the dynamic dipolar field. The upper branch corresponds to the mode for which facing edges oscillate in phase, leading to intensification of the dynamic dipolar field. This explains the large gap between the branches at $k_{z}=0$. These nonuniform modes may be excited by a pulsed field that is uniform within the plane because a nonuniform ground state results from antiferromagnetic ordering of the array. When the array is saturated along its length and a uniform ground state is obtained, only a mode that is uniform within the plane may be excited by a pulsed field that is uniform within the plane, as shown in Fig. 9(j).

While dynamic dipolar interactions explain the splitting of the modes at $k_{z}=0$, the relatively high frequencies of the branches observed at $k_{z}=0$ for small field values require further comment. Indeed, comparing Figs. 9(e) and 9(f) one can see that the bottom of the parabolic branch at zero field and at saturation has similar frequency. Attenuation of the dynamic dipolar field at zero field (as the facing edges of nearest-neighbor nanowires oscillate out of phase) might be expected to push the frequency of the bottom of the parabola to a lower value compared to that at the saturation field (where the mode profile is uniform within the plane of the array), since the $z$ component of the static internal mean field (the sum of the applied and demagnetizing fields) does not change and remains about zero for fields less than the saturation value (see Fig. 8). Furthermore, the frequency of the upper branch at $k_{z}=0$ for zero applied field might be expected to be comparable to the frequency of the single branch at $k_{z}=0$ at the saturation field, since in both cases facing nanowire edges oscillate in phase. The high-frequency results from the influence of the exchange interaction upon the resonant modes, which are nonuniform within the plane of the array as a result of the antiferromagnetic ordering. As the field increases towards the saturation value, the mode profile changes from nonuniform to uniform within the plane of the array, reducing the influence of the exchange interaction and leading to a decrease of frequency.

Comparing the experimental and simulated resonance frequencies at low fields [Figs. 7(a), 7(b), and 7(d)] the experimental frequencies are seen to be somewhat higher. This may indicate that the exchange interaction plays a more significant role in the presence of structural disorder.

Figure 10(a) shows simulated spectra for the array with $d=$ $85 \mathrm{~nm}$ and $p=0.66$ in response to an excitation applied to only the top $50 \mathrm{~nm}$ of the array. The response of 50-nm-long regions located at the top, middle, and bottom of the array is shown. Comparing the relative amplitude of the response of the different sections, the dominant peaks are seen to be associated with $\mathrm{E}$ modes localized within demagnetizing wells both at the top and bottom ends of the nanowires. End excitations penetrate through the entire length of the nanowire with small amplitude not visible in Fig. 10(a) and appear at the other end with amplitude comparable to that at the top surface, where the excitation was applied. A small damping constant of $\alpha=$ 0.005 was assumed so as to attenuate the spin waves only slightly. Propagating spin waves form a continuous spectrum, and the propagating modes are excited with one order of magnitude smaller amplitude than that of the E modes and are not visible within the presented spectra. The frequencies of the E modes are very low and almost independent of field below the saturation value. Above saturation their frequencies increase with the field, as expected from the internal field profile shown in Fig. 8.

Figure 10(b) shows simulated spectra for a single wire with $d=85 \mathrm{~nm}$ in response to a pulse applied to the top $50 \mathrm{~nm}$ of its length. Again, as in Fig. 10(a), the response of 50-nm-long regions located at the top, middle, and bottom of the nanowire is shown. These spectra are qualitatively different from those 

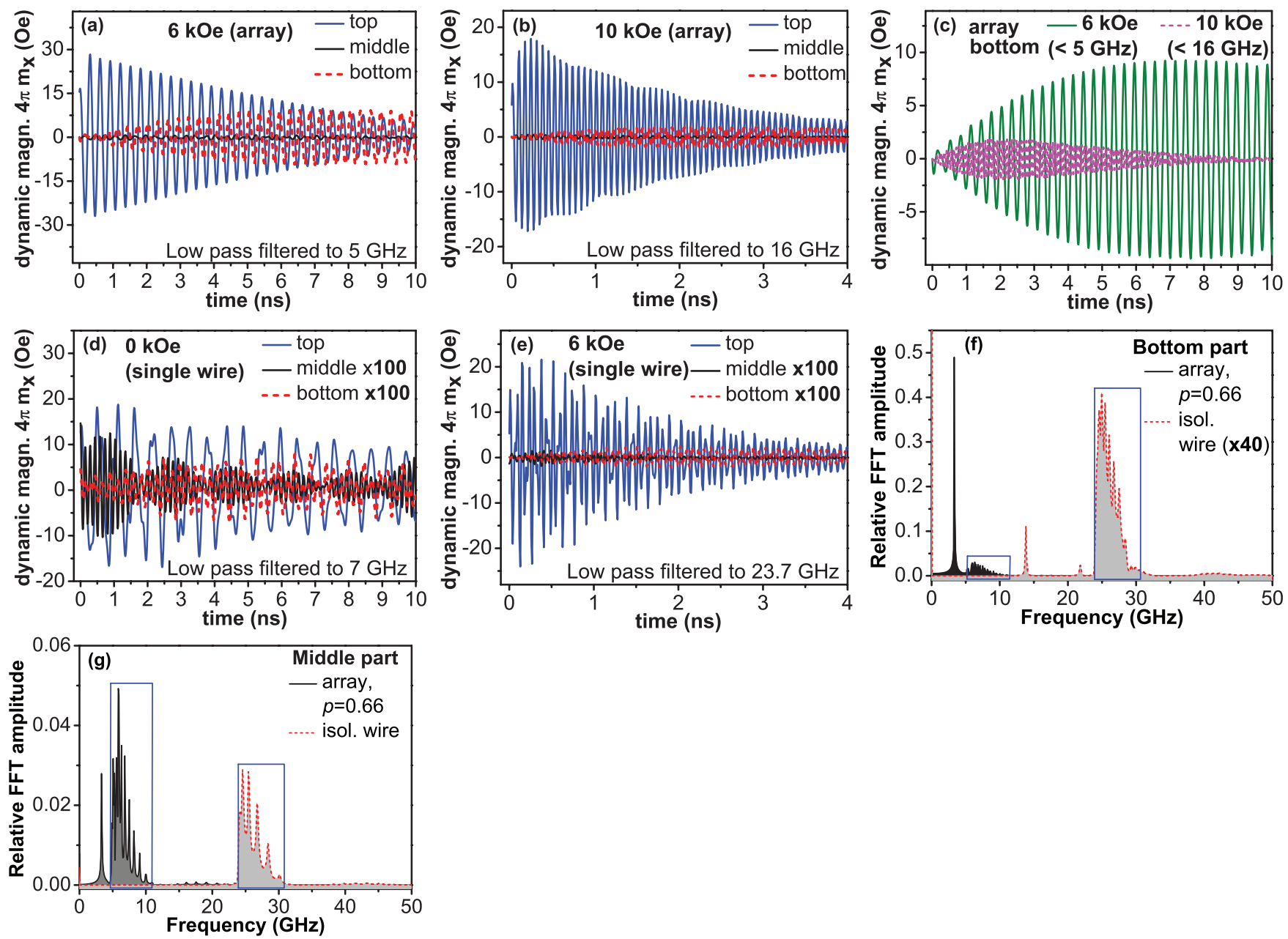

FIG. 11. (Color online) Component of the averaged dynamic magnetization, which is perpendicular to both the nanowire axis and the excitation pulse, in response to a pulsed field applied to only the top $50 \mathrm{~nm}$ of the array for (a) an array with $p=0.66$ and $H=6 \mathrm{kOe}$ applied field; (b) an array with $p=0.66$ and $H=10 \mathrm{kOe}$; (d) a single wire for $H=0 \mathrm{kOe}$; (e) a single wire for $H=6 \mathrm{kOe}$. The blue and black solid lines and red dashed line correspond to the averaged dynamic magnetization of 50-nm-long regions at the top, middle, and bottom of the nanowires, respectively. (c) Comparison of averaged dynamic magnetization of 50-nm-long regions at the bottom of the array corresponding to the red dashed curves in (a) and (b). The signals are low pass filtered with the cutoff frequency indicated for the signals within the panel. Spectra acquired from the bottom (f) and middle (g) $50 \mathrm{~nm}$ of the array with $p=0.66$ (black solid line) and isolated wire (red dashed line), normalized to the height of the largest peak (which corresponds to an end mode) in the spectrum acquired from the top $50 \mathrm{~nm}$ of the array and isolated wire, respectively. The bias field was $6 \mathrm{kOe}$, and the boxes indicate the continuous spectrum of propagating spin waves.

obtained from the arrays. First, higher order E modes are clearly observed in the spectrum from the excited region. Second, unlike for the array with $p=0.66$, the amplitude of the E modes localized at the other end of the nanowires is one order of magnitude smaller compared to that of the $\mathrm{E}$ modes localized at the top surface, where the excitation was applied and, hence, not visible in the spectrum. As for the array, the propagating modes are excited with one order of magnitude smaller amplitude than the E modes, and to make them visible in the spectra the responses of the middle part are magnified by a factor of 10 and that of the bottom part are magnified by a factor indicated separately for each panel.

Simulated spectra for arrays with $d=85 \mathrm{~nm}$ and different packing densities $p$ in response to a pulse applied to the top $50 \mathrm{~nm}$ of the nanowires are compared in Fig. 10(c). The frequencies of the $\mathrm{E}$ modes do not depend on $p$ at remanence. However, as the field increases a pronounced dependence on $p$ develops, the frequency decreasing as $p$ increases. Figure 10(d) shows simulated spectra for the same arrays as the full volume of the array is excited and sensed. The frequencies predicted by Eq. (2) are also indicated and are seen to describe the frequencies of the fundamental D modes well, both above and slightly below saturation for all $p$ values. For the single nanowire, the frequency calculated from Eq. (2) reproduces the simulated frequency exactly and so was not indicated in the figure for the sake of clarity. The frequencies of the D modes decrease significantly with increasing $p$ at high field due to the dipolar interactions between the nanowires. At remanence this dependence is very weak because the decrease of the frequency due to dipolar interactions is compensated by the influence of the exchange interaction associated with the excitation of modes that are nonuniform within the plane of the array, as was described previously. 
Figure 11 compares the simulated dynamic magnetization in response to a pulse applied to only the top $50 \mathrm{~nm}$ of the array with $d=85 \mathrm{~nm}$ and $p=0.66$ and a single wire with $d=85 \mathrm{~nm}$. The component of the simulated dynamic magnetization perpendicular to both the nanowire axis and the excitation pulse averaged over 50-nm-long regions located at the top, middle, and bottom of the array and single wire is shown. The signals are low pass filtered with the cutoff frequency selected in each panel so as to isolate response due to the E modes, which were observed to dominate the response in Fig. 10. In the case of the array, we observe that the spin-wave energy is transferred from end excitations localized at the top of the array, where the localized excitation pulse is applied, to the end excitations localized at the bottom of the array. This leads to a significant increase of the dynamic magnetization amplitude at the bottom of the array with increasing time, which exceeds the dynamic amplitude for the central part and even eventually exceeds the dynamic amplitude for the top part of the nanowires [Fig. 11(a)].

Comparing Figs. 11(a) and 11(b), the increase of the dynamic magnetization amplitude at the bottom of the array relative to that at the top is larger for smaller applied field values. The characteristic decay time of the magnetization oscillations is inversely proportional to the internal field, so that there is less time available for transfer of energy from one end of the nanowire to the other for larger applied field values. Yet, as can be seen from Fig. 11(c), the increase of the dynamic magnetization amplitude at the bottom of the array occurs about 3 times faster when the field is increased from 6 to $10 \mathrm{kOe}$. As can be seen from Figs. 11(d) and 11(e), the dynamic magnetization amplitude at the bottom end of the single nanowire remains negligible compared to the dynamic magnetization amplitude at the top end of the single nanowire. This indicates that the observed energy transfer between the end modes localized at the top and bottom surfaces of the array has a significantly reduced efficiency for a single nanowire.

The observed energy transfer between end modes should be distinguished from the transfer of energy by propagating spin waves, e.g., observed in micromagnetic simulations for a single nanowire reported in Ref. 67. The propagating spin waves are also observed in the present case both for the array and the single nanowire. However, unlike the case of the end excitations, the amplitude of traveling spin waves is comparable at the bottom and top of both the single nanowire and the nanowires within an array, thanks to the reduced damping used in the simulations and the relatively short length of the nanowires.

As can be seen from Fig. 11(f), the experiment proposed in Ref. 67 and performed with the present samples could actually be dominated by the response due to the more easily excited end modes rather than the propagating modes. It should also be noted that increasing either the damping or the length of the nanowires would weaken the effect of energy transfer between the end modes. The energy transfer between end modes is significantly more efficient in the case of the array as compared to the isolated wire. The difference could be related to two factors. First, the range of the dynamic dipolar field produced by the E modes at the end of each wire is greater when such wires form an array and increases as the array's packing density increases. Second, the demagnetizing field in the array modifies the depth of the demagnetized wells and the spectrum and spatial character of the localized modes as well as the character of the frequency dispersion of propagating spin waves. The frequencies of the localized modes are located below the lowest frequency of the continuum spectrum of propagating modes. The energy transfer could therefore be considered as tunneling of the localized modes through the middle part of the wire. The tunneling (and associated energy transfer) is more efficient for modes located closer to the top of the "barrier." From Fig. 9, the frequency separation of the end modes from the continuum spectrum at $k_{z}=0$ is significantly smaller for the arrays. Figure $11(\mathrm{~g})$ shows the difference in tunneling of the end excitations through the middle part of the nanowires. In the middle part of the array, the height of the peak attributed to the end mode is comparable to the height of the peaks within the continuous spectrum. In the middle part of the isolated nanowire, the peak attributed to the end mode is indistinguishably small in comparison with the continuous spectrum. This discussion shows how the properties of an array cannot necessarily be inferred from those of a single nanowire.

The localization of modes of different frequency also provides other opportunities. For example, nanowires could be functionalized with organic coatings so as to selectively target certain biological tissues. By tuning the frequency of an applied ac magnetic field, the selective excitation of $\mathrm{E}$ or $\mathrm{D}$ modes could then be used as a highly localized source of heating.

\section{B. Perpendicular field geometry}

Experimental spectra obtained from TRMOKE measurements in the perpendicular field geometry (in which the bias field is applied perpendicular to the length of the wires and therefore in the plane of the arrays) from the arrays with nominal $p=0.38$ and 0.41 are shown in Figs. 12(a) and 12 (b), respectively. The spectra for $p=0.38$ are much more noisy than those for $p=0.41$. This may be related to the greater incidence of missing nanowires evident in the SEM images of Fig. 1 and the somewhat larger nanowire diameter. The difference in the quality of the spectra is more significant in the perpendicular as opposed to the parallel geometry. This is because defects and disorder in the array lead to more significant nonuniformity of the internal field when the magnetization has a component perpendicular to the length of the nanowires. This has important implications for applications, since it is very expensive and time consuming to produce highly ordered arrays.

The frequency of the prominent peak in the spectra shown in Fig. 12(b) appears almost independent of field below the saturation value of $2.4 \mathrm{kOe}$ and up to the maximum value of $3.5 \mathrm{kOe}$. Again this may be the result of the disorder within the array. By analogy with Eq. (2), a simple phenomenological equation may be constructed for the frequency of the fundamental mode above saturation when the array is magnetized perpendicular to the length of the nanowires: ${ }^{20}$

$$
f=\gamma\{\mathrm{H}[\mathrm{H}+2 \pi(3 p-1) \mathrm{M}]\}^{1 / 2} .
$$

Equation (3) gives the exact result in the limiting cases of a single wire magnetized perpendicular to its length $(p=0)$ and a continuous film magnetized in plane $(p=1)$. The frequencies 

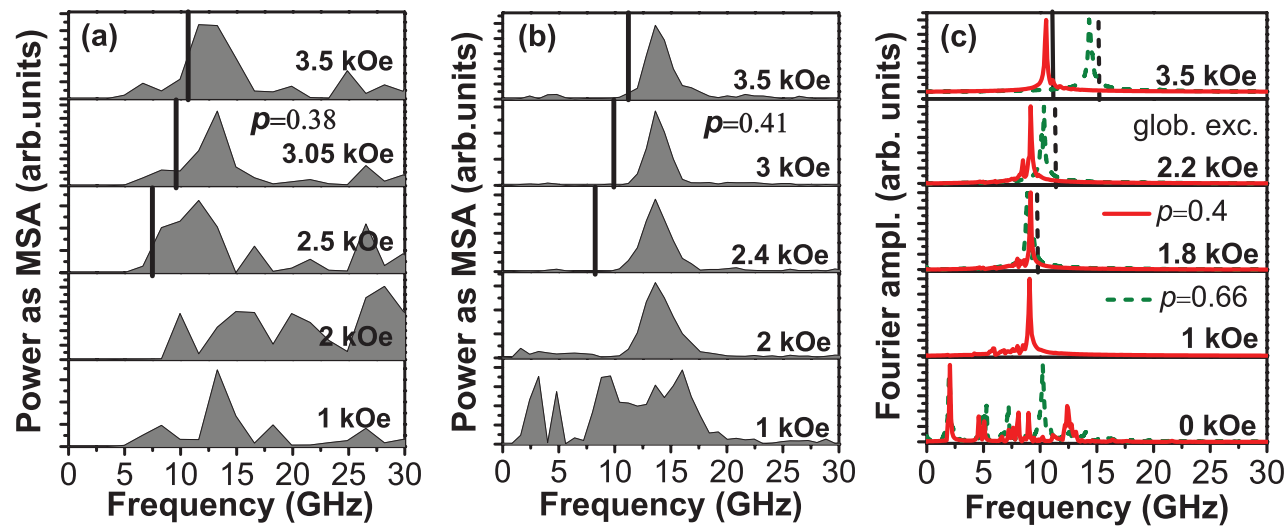

FIG. 12. (Color online) (a),(b) Spectra obtained from TRMOKE measurements of the arrays with $p=0.38$ and 0.41 , respectively. Black solid vertical lines indicate frequencies calculated from Eq. (3). (c) Simulated spectra for an array with $d=85 \mathrm{~nm}$ and $p=0.4$ (solid red line) and 0.66 (dashed green line), for which the full volume of the array was excited and sensed. Black solid and dashed vertical lines indicate frequencies calculated from Eq. (3) for $p=0.4$ and 0.66 , respectively.

calculated from Eq. (3) are indicated by black vertical lines within Figs. 12(a) and 12(b) and seen to lie somewhat below the experimental values.

As for the parallel geometry, simulations were performed with the pulse applied to either the full length or the top 50-nm section of the nanowire array. In contrast to the results for the parallel geometry, Fig. 12(c) shows that the field dependence of the frequency exhibits a qualitative change as the packing density is varied. For $p=0.4$, the frequency of the dominant peak within the spectrum is almost independent of field from $1 \mathrm{kOe}$ up to saturation at $3.1 \mathrm{kOe}$, while for $p=0.66$ the frequency varies with applied field both below and above saturation. The frequencies predicted by Eq. (3) are shown for fields above saturation and, unlike in the experiment, describe the simulated frequencies very well. The experiment and simulations for $p=0.4$ yield the same qualitative behavior below saturation, but the experimental frequencies are larger by a factor of about 1.5 . This discrepancy is again attributed to defects and disorder within the real array, which significantly influence the internal field in the perpendicular geometry.

The different behavior observed for $p=0.4$ and 0.66 in Fig. 12(c) can be explained by considering the component of internal field in the direction of the applied field, which is presented in Figs. 13(a) and 13(b). The field was applied along a line connecting the centers of the two nanowires within the unit cell. For $p=0.4$, this internal field has a flat profile and remains close to zero up to saturation, while for $p=0.66$ the internal field increases at the edges of the nanowires, leading to an increase of the mean internal field and resonance frequencies.

Simulated spectra for the array with $d=85 \mathrm{~nm}$ and $p=0.66$ in the perpendicular field geometry in response to a pulse applied to only the top $50 \mathrm{~nm}$ of the array are shown in Fig. 14(a). As discussed previously, the dominant peaks at zero field correspond to edge modes localized in demagnetizing wells on the top and bottom surfaces of the array. The largest peak at a field of $1 \mathrm{kOe}$ also corresponds to the mode localized at the top of the array. When the field is increased further, the relative amplitude of precession within the middle part of the nanowires increases and above saturation becomes comparable with the precession amplitude within the top part. This happens because the demagnetized regions at the top and bottom become smaller, so that a larger proportion of the energy from the applied pulse is transferred to spin-wave modes propagating along the nanowire. The effect of the pulse applied to the top section of the array is therefore qualitatively
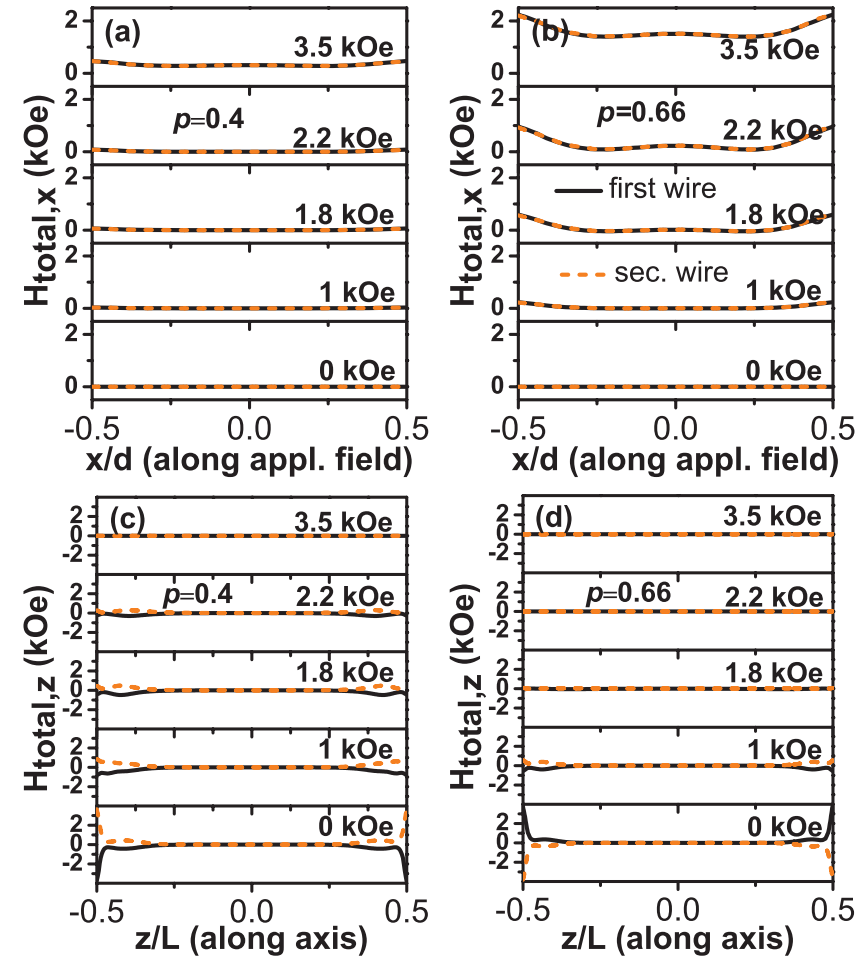

FIG. 13. (Color online) (a),(b) Component of total internal field (sum of applied and demagnetizing fields) in the direction of the applied field in two neighboring nanowires that have antiparallel magnetization at remanence as a function of the position along a line parallel to the applied field that passes through the center of the nanowires, for $p=0.4$ and 0.66 , respectively. (c),(d) The component of total internal field parallel to the symmetry axes of the same nanowires as a function of position on the nanowire axis for $p=$ 0.4 and 0.66 , respectively. 

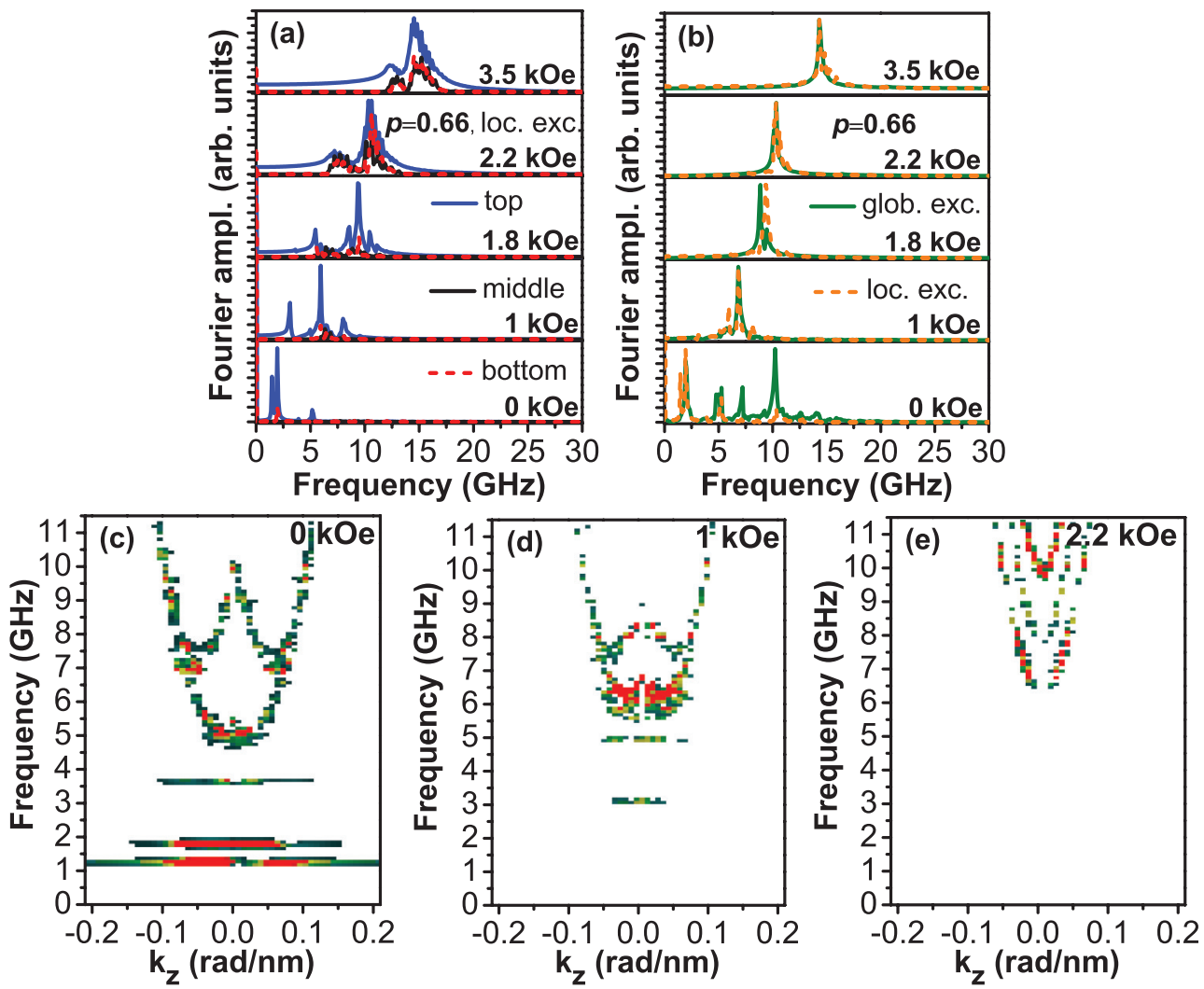

FIG. 14. (Color online) (a) Simulated spectra for an array with $d=85 \mathrm{~nm}$ and $p=0.66$ in the perpendicular field geometry in response to a pulse applied to only the top $50 \mathrm{~nm}$ of the array. The blue and black solid lines and red dashed line correspond to the response of 50-nm-long sections at the top, middle, and bottom of the array, respectively. (b) Simulated spectra for the response of the full volume of the same array, in the perpendicular field geometry, to a pulse applied to the full volume (solid green line) and only the top $50 \mathrm{~nm}$ (orange dashed line) of the array. (c),(d),(e) Dispersion curves for spin waves propagating along the nanowires for applied fields of 0, 1, and $2.2 \mathrm{kOe}$, respectively.

different in the parallel and perpendicular field geometries. In the former case energy was transferred mostly into localized end modes for all values of the static applied field.

Simulated spectra obtained by sensing the full volume of the array with the pulse applied to the top $50 \mathrm{~nm}$ and the full volume of the array are compared within Fig. 14(b). Dispersion curves for spin waves propagating along the nanowires are shown in Figs. 14(c)-14(e) for applied fields of 0, 1, and $2.2 \mathrm{kOe}$, respectively. From Fig. 14(b), the frequency of the dominant peak in the spectrum at $1 \mathrm{kOe}$ applied field $(6.8 \mathrm{GHz})$ is seen to be lower than the highest frequency of the dominant peaks at $0 \mathrm{kOe}(10.2 \mathrm{GHz})$. Both peaks correspond to delocalized modes, which appear to belong to different branches of the dispersion relation. As can be seen from Figs. 14(c) and 14(d), the splitting of the two branches at $k_{z}=0$ becomes smaller as the applied field is increased. At $1.8 \mathrm{kOe}$ the dominant peaks excited by the localized and uniformly applied pulse are slightly shifted from one another. The peaks are associated with different branches of the dispersion curve that have almost merged as saturation is approached. Figure 14(e) shows three branches for applied fields above saturation, each of which has positive group velocity. The dominant peak, which is excited by both localized and uniformly applied pulses, corresponds to the branch with the highest frequency, which originates from the fundamental uniform mode of a single wire. The two lower frequency branches originate from modes localized on opposite sides of the cross section of the nanowires along the applied magnetic field.

\section{CONCLUSIONS}

The static and dynamic properties of MNWAs with high packing densities ( $p=0.4$ to 0.66 ) have been studied both experimentally and by micromagnetic simulation. The competition between anisotropies associated with the shape of the individual nanowires and the array as a whole significantly modifies both the static and dynamical properties of the array compared to the limiting cases of a single nanowire and a continuous ferromagnetic film. For high packing densities, the easy-plane shape anisotropy of the array overcomes the easy-axis shape anisotropy of the individual nanowires. The resulting magnetic configuration yields zero net magnetization in directions orthogonal to that of the applied field, and zero remanence irrespective of the field history. At remanence individual nanowires are magnetized along their length but in an antiparallel configuration within the array. Micromagnetic simulations show that the demagnetizing field arising from the top and bottom surfaces of the entire array compensates for the external field applied parallel to the nanowires up to the saturation value. This leads to a mean internal field that remains approximately equal to zero in the middle of the wires 
as the applied field is increased up to the saturation value and which only begins to grow as the saturation value is exceeded.

The spectra of excitations obtained from experiment and micromagnetic simulation are in qualitative agreement when the external magnetic field is applied either parallel or perpendicular to the symmetry axes of the nanowires. For the parallel field geometry, there is also good quantitative agreement between experiment and simulation. The mode frequencies were observed to initially decrease as the field was increased from remanence towards the saturation value. This was shown to result from a change of the mode character within the plane of the array from nonuniform to uniform as the ground state changed with field. Quantitative differences between experimental and simulated spectra in the perpendicular field geometry were attributed to the presence of structural disorder within the array.

The dependence of the frequency of both end and delocalized modes upon packing density was investigated. The frequencies of both types of modes are found to depend only weakly upon packing density at remanence, while a qualitatively different behavior is observed in the parallel and perpendicular field geometries as the field is increased. In the parallel field geometry, a significant decrease of frequency for both types of mode is observed with increasing $p$ at high field. In the perpendicular field geometry, a qualitative change is observed as the packing density is varied. For $p=0.4$, the frequency of the dominant peak within the spectrum is almost independent of field from $1 \mathrm{kOe}$ up to saturation at $3.1 \mathrm{kOe}$, while for $p=0.66$ the frequency varies with applied field both below and above saturation. The robust ground state and frequencies observed at resonance are attractive for the construction of microwave devices that can operate without an external bias magnetic field.

Finally, we observed that the localized end modes could be detected at the bottom of the structure following localized excitation of the top. This effect could be described as tunneling of the end modes across the middle of the wire. The tunneling is observed to be very efficient in densely packed arrays, as a result of the extended penetration of the dynamic demagnetizing fields into the middle of the wires and also of the lowering of the tunnel barrier by the static demagnetizing field in the array. This result could be useful in the construction of optically activated magnonic devices.

\section{ACKNOWLEDGMENTS}

The authors gratefully acknowledge the assistance of V.-A. Antohe and S. Tuilard with sample fabrication and M. Dvornik, M. Franchin, and H. Fangohr with micromagnetic simulations. The financial support from the European Community's Seventh Framework Programme (FP7/2007-2013) under Grant Agreements No. 212257 MASTER (fabrication and experiment) and No. 233552 DYNAMAG (simulations) is gratefully acknowledged. We also gratefully acknowledge financial support from a UKIERI-DST standard research award (Grants No. SA 07-021 and No. DST/INT/UKIERI/SA/P2/2008) for travel between S. N. B. N. C. B. S., India, and the University of Exeter, United Kingdom. Finally, V.V.K. gratefully acknowledges funding received from the U.K. Engineering and Physical Sciences Research Council Project No. EP/E055087/1.

\footnotetext{
*r.j.hicken@exeter.ac.uk

${ }^{1}$ D. Routkevitch, A. A. Tager, J. Haruyama, D. Almawlawi, M. Moskovits, and J. M. Xu, IEEE Trans. Electron Devices 43, 1646 (1996).

${ }^{2}$ J. I. Martin, J. Nogues, K. Liuc, J. L. Vicent, and I. K. Schuller, J. Magn. Magn. Mater. 256, 449 (2003).

${ }^{3}$ T. M. Whitney, P. C. Searson, J. S. Jiang, and C. L. Chien, Science 261, 1316 (1993).

${ }^{4}$ B. Cui, W. Wu, L. Kong, X. Sun, and S. Y. Chou, J. Appl. Phys. 85, 5534 (1999).

${ }^{5}$ C. Ross, Annu. Rev. Mater. Res. 31, 203 (2001).

${ }^{6}$ M. Darques, J. Spiegel, J. Dela Torre Medina, I. Huynen, and

L. Piraux, J. Magn. Magn. Mater. 321, 2055 (2009).

${ }^{7}$ L.-P. Carignan, A. Yelon, D. Menard, and C. Caloz, IEEE Trans. Microwave Theory Tech. 59, 2568 (2011).

${ }^{8}$ C. Caloz, L.-P. Carignan, V. Boucher, T. Kodera, S. Couture, A. Parsa, D. Menard, and A. Yelon, IEEE MTT-S International Microwave Symposium (IMS), Anaheim, CA (IEEE, New York, 2010), p. 1416.

${ }^{9}$ M. Darques, J. Dela Torre Medina, L. Piraux, L. Cagnon, and I. Huynen, Nanotechnology 21, 145208 (2010).

${ }^{10}$ D. H. Reich, M. Tanase, A. Hultgren, L. A. Bauer, C. S. Chen, and G. J. Meyer, J. Appl. Phys. 93, 7275 (2003).

${ }^{11}$ R. Singh and H. S. Nalwa, J. Biomed. Nanotech. 7, 489 (2011).

${ }^{12}$ T. G. Sorop, C. Untiedt, F. Luis, M. Kroll, M. Ras, and L. J. de Jongh, Phys. Rev. B 67, 014402 (2003).
}

${ }^{13}$ C. A. Ross, M. Hwang, M. Shima, J. Y. Cheng, M. Farhoud, T. A Savas, Henry I. Smith, W. Schwarzacher, F. M. Ross, M. Redjdal, and F. B. Humphrey, Phys. Rev. B 65, 144417 (2002).

${ }^{14}$ H. Zeng, R. Skomski, L. Menon, Y. Liu, S. Bandyopadhyay, and D. J. Sellmyer, Phys. Rev. B 65, 134426 (2002).

${ }^{15}$ X. Y. Zhang, G. H. Wen, Y. F. Chan, R. K. Zheng, X. X. Zhang, and N. Wang, Appl. Phys. Lett. 83, 3341 (2003).

${ }^{16}$ W. Li, Y. Peng, J. Zhang, G. A. Jones, and T. H. Shen, J. Phys.: Conf. Ser. 17, 20 (2005).

${ }^{17}$ Y. Peng, T.-H. Shen, and B. Ashworth, J. Appl. Phys. 93, 7050 (2003).

${ }^{18}$ Y. Peng, T.-H. Shen, B. Ashworth, X.-G. Zhao, C. A. Faunce, and Y.-W. Liu, Appl. Phys. Lett. 83, 362 (2003).

${ }^{19}$ A. Encinas-Oropesa, M. Demand, L. Piraux, I. Huynen, and U. Ebels, Phys. Rev. B 63, 104415 (2001).

${ }^{20} \mathrm{M}$. Demand, A. Encinas-Oropesa, S. Kenane, U. Ebels, I. Huynen, and L. Piraux, J. Magn. Magn. Mater. 249, 228 (2002).

${ }^{21}$ A. Encinas-Oropesa, M. Demand, L. Piraux, U. Ebels, and I. Huynen, J. Appl. Phys. 89, 6704 (2001).

${ }^{22}$ A. Sklyuyev, M. Ciureanu, C. Akyel, P. Ciureanu, and A. Yelon, J. Appl. Phys. 105, 023914 (2009).

${ }^{23}$ V. Boucher, C. Lacroix, L.-P. Carignan, A. Yelon, and D. Ménard, Appl. Phys. Lett. 98, 112502 (2011).

${ }^{24}$ T. M. Nguyen, M. G. Cottam, H. Y. Liu, Z. K. Wang, S. C. Ng, M. H. Kuok, D. J. Lockwood, K. Nielsch, U. Gösele, Phys. Rev. B 73, 140402(R) (2006). 
${ }^{25}$ A. A. Stashkevich, Y. Roussigné, P. Djemia, S. M. Chérif, P. R. Evans, A. P. Murphy, W. R. Hendren, R. Atkinson, R. J. Pollard, A. V. Zayats, G. Chaboussant, and F. Ott, Phys. Rev. B 80, 144406 (2009).

${ }^{26}$ R. Arias and D. L. Mills, Phys. Rev. B 63, 134439 (2001); 66, 149903(E) (2002).

${ }^{27}$ R. Arias and D. L. Mills, Phys. Rev. B 70, 094414 (2004).

${ }^{28}$ E. V. Tartakovskaya, Phys. Rev. B 71, 180404(R) (2005).

${ }^{29}$ R. Arias and D. L. Mills, Phys. Rev. B 72, 104418 (2005).

${ }^{30}$ R. Arias, Physica B 384, 25 (2006).

${ }^{31}$ E. V. Tartakovskaya, Physica B 385, 468 (2006).

${ }^{32}$ R. Arias and D. L. Mills, Phys. Rev. B 67, 094423 (2003).

${ }^{33}$ V. E. Kireev, R. S. Khymyn, B. A. Ivanov, C. E. Zaspel, arXiv:1201.1747.

${ }^{34}$ J. De La Torre Medina, M. Darques, L. Piraux, and A. Encinas, J. Appl. Phys. 105, 023909 (2009).

${ }^{35}$ L. Sampaio, E. H. C. P. Sinnecker, G. R. C. Cernicchiaro, M. Knobel, M. Vazquez, and J. Velazquez, Phys. Rev. B 61, 8976 (2000).

${ }^{36}$ C. C. Dantas, Physica E 44, 675 (2011).

${ }^{37}$ F. Zighem, T. Maurer, F. Ott, and G. Chaboussant, J. Appl. Phys. 109, 013910 (2011).

${ }^{38}$ J. Velazquez, C. Garcia, M. Vazquez, and A. Hernando, J. Appl. Phys. 85, 2768 (1999).

${ }^{39}$ R. Engel-Herbert, T. Hesjedal, J. Mohanty, D. M. Schaadt, and K. H. Ploog, Phys. Rev. B 73, 104441 (2006).

${ }^{40}$ J. Ding, M. Kostylev, and A. O. Adeyeye, Phys. Rev. Lett. 107, 047205 (2011).

${ }^{41}$ J. Ding, M. Kostylev, and A. O. Adeyeye, Phys. Rev. B 84, 054425 (2011).

${ }^{42}$ A. Y. Galkin, B. A. Ivanov, and C. E. Zaspel, Phys. Rev. B 74, 144419 (2006).

${ }^{43}$ M. Dvornik, P. V. Bondarenko, B. A. Ivanov, and V. V. Kruglyak, J. Appl. Phys. 109, 07B912 (2011).

${ }^{44}$ H. Puszkarski, M. Krawczyk, and J. C. S. Levy, J. Appl. Phys. 101, 024326 (2007).

${ }^{45}$ H. Puszkarski, M. Krawczyk, and H. T. Diep, Surf. Sci. 602, 2197 (2008).

${ }^{46}$ L.-P. Carignan, C. Lacroix, A. Ouimet, M. Ciureanu, A. Yelon, and D. Ménard, J. Appl. Phys. 102, 023905 (2007).

${ }^{47}$ M. Hwang, M. C. Abraham, T. A. Savas, H. I. Smith, R. J. Ram, and C. A. Ross, J. Appl. Phys. 87, 5108 (2000).

${ }^{48}$ T. Fischbacher, M. Franchin, G. Bordignon, and H. Fangohr, IEEE Trans. Magn. 43, 2896 (2007); User manual is available at http://nmag.soton.ac.uk/nmag/current/manual/html/manual.html
${ }^{49}$ L. Piraux, K. Renard, R. Guillemet, S. Mátéfi-Tempfli, M. MátéfiTempfli, V. Antohe, S. Fusil, K. Bouzehouane, and V. Cros, Nano Lett. 7, 2563 (2007).

${ }^{50}$ S. Mátéfi-Tempfli and M. Mátéfi Tempfli, Adv. Mater. 21, 4005 (2009).

${ }^{51}$ P. Biagioni, M. Celebrano, M. Savoini, G. Grancini, D. Brida, S. Mátéfi-Tempfli, M. Mátéfi-Tempfli, L. Duò, B. Hecht, G. Cerullo, and M. Finazzi, Phys. Rev. B 80, 045411 (2009).

${ }^{52}$ S. Habouti, M. Mátéfi-Tempfli, C.-H. Solterbeck, M. Es-Souni, S. Mátéfi-Tempfli, and M. Es-Souni, Nano Today 6, 12 (2011).

${ }^{53}$ Y. Liu, L. R. Shelford, V. V. Kruglyak, R. J. Hicken, Y. Sakuraba, M. Oogane, and Y. Ando, Phys. Rev. B 81, 094402 (2010).

${ }^{54}$ R. Wilks, N. D. Hughes, and R. J. Hicken, J. Appl. Phys. 91, 8670 (2002).

${ }^{55}$ R. Wilks, R. J. Hicken, M. Ali, B. J. Hickey, A. T. G. Pym, J. D. R. Buchanan, and B. K. Tanner, J. Appl. Phys. 95, 7441 (2004).

${ }^{56}$ M. van Kampen, C. Jozsa, J. T. Kohlhepp, P. LeClair, L. Lagae, W. J. M. de Jonge, and B. Koopmans, Phys. Rev. Lett. 88, 227201 (2002).

${ }^{57}$ A. Barman, S. Wang, S. Wang, J. D. Maas, A. R. Hawkins, S. Kwon, A. Liddle, J. Bokor, and H. Schmidt, Nano Lett. 6, 2939 (2006).

${ }^{58}$ G. M. Müller, G. Eilers, Z. Wang, M. Scherff, R. Ji, K. Nielsch, C. A. Ross, and M. Münzenberg, New J. Phys. 10, 123004 (2008).

${ }^{59}$ S. Pal, B. Rana, O. Hellwig, T. Thomson, and A. Barman, Appl. Phys. Lett. 98, 082501 (2011).

${ }^{60}$ J. D. Jackson, Classical Electrodynamics, 3rd ed. (Wiley, Singapore, 1999).

${ }^{61}$ H. Fangohr, G. Bordignon, M. Franchin, A. Knittel, P. A. J. de Groot, and T. Fischbacher, J. Appl. Phys. 105, 07 D529 (2009).

${ }^{62}$ O. Dmytriiev, T. Meitzler, E. Bankowski, A. Slavin, and V. Tiberkevich, J. Phys.: Condens. Matter 22, 136001 (2010).

${ }^{63}$ O. Dmytriiev, M. Dvornik, R. V. Mikhaylovskiy, M. Franchin, H. Fangohr, L. Giovannini, F. Montoncello, D. V. Berkov, E. K. Semenova, N. L. Gorn, A. Prabhakar, and V. V. Kruglyak, Phys. Rev. B 86, 104405 (2012).

${ }^{64}$ M. Dvornik, Y. Au, and V. V. Kruglyak, Top. Appl. Phys. 125, 101 (2013).

${ }^{65}$ L.-P. Carignan, M. Massicotte, C. Caloz, A. Yelon, D. Ménard, IEEE Trans. Magn. 45, 4070 (2009).

${ }^{66}$ The dispersion curves and mode profiles were calculated using SEMARGL (http://www.magnonics.org/semargl/); M. Dvornik and V. V. Kruglyak, Phys. Rev. B 84, 140405 (2011).

${ }^{67}$ V. V. Kruglyak and R. J. Hicken, J. Magn. Magn. Mater. 306, 191 (2006). 